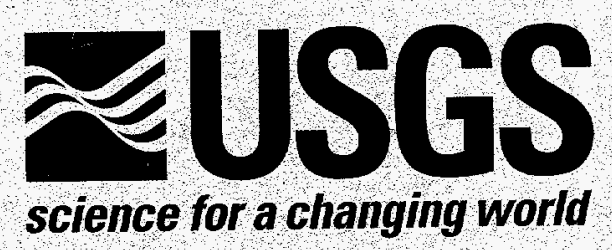

\title{
HYDROCHEMICAL INVESTIGATIONS IN
}

CHARACTERIZING THE UNSATURATED ZONE AT YUCCA MOUNTAIN, NEVADA

\section{USGS/WRIR- $-98-4132$}

\section{U.S. GEOLOGICAL SURVEY}

Water-Resources Investigations Report 98-4132

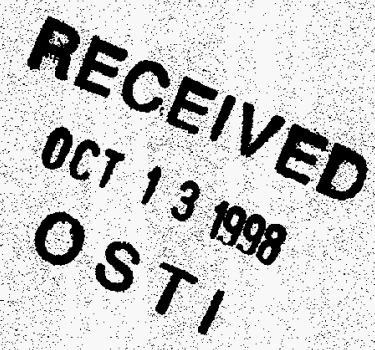

Prepared in cooperation with the NEVADA OPERATIONS OFFICE, U.S. DEPARTMENT OF ENERGY, under Interagency Agreement DE-AI08-97NV12033

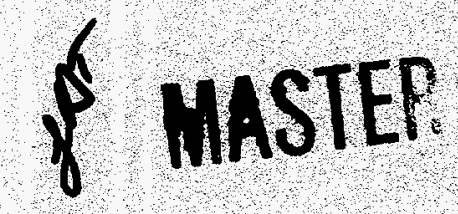




\section{Hydrochemical Investigations in Characterizing the Unsaturated Zone at Yucca Mountain, Nevada}

By In C. Yang, ${ }^{1}$ Pei Yu, ${ }^{2}$ Gordon W. Rattray, ${ }^{1}$ Janine S. Ferarese, ${ }^{1}$ and Joseph N. Ryan ${ }^{3}$

${ }^{1}$ U.S. Geological Survey, Denver, Colorado

${ }^{2}$ Taipower Company, Taipei, Taiwan

${ }^{3}$ Department of Civil, Environmental, and Architectural Engineering, University of Colorado, Boulder, Colorado

\section{U.S. GEOLOGICAL SURVEY}

Water-Resources Investigations Report 98-4132

Prepared in cooperation with the

NEVADA OPERATIONS OFFICE, U.S. DEPARTMENT OF ENERGY under

INTERAGENCY AGREEMENT DE-AI08-97NV12033 


\title{
U.S. DEPARTMENT OF THE INTERIOR BRUCE BABBITT, Secretary
}

\author{
U.S. GEOLOGICAL SURVEY
}

Thomas J. Casadevall, Acting Director

The use of firm, trade, and brand names in this report is for identification purposes only and does not constitute endorsement by the U.S. Geological Survey.

For additional information write to:

Chief, Earth Science Investigations Program

Yucca Mountain Project Branch

U.S. Geological Survey

Box 25046, Mail Stop 421

Denver Federal Center

Denver, CO 80225-0046
Copies of this report can be purchased from:

U.S. Geological Survey

Information Services

Box 25286

Denver, CO 80225-0286 


\section{DISCLAIMER}

This report was prepared as an account of work sponsored by an agency of the United States Government. Neither the United States Government nor any agency thereof, nor any of their employees, makes any warranty, express or implied, or assumes any legal liability or responsibility for the accuracy, completeness, or usefulness of any information, apparatus, product, or process disclosed, or represents that its use would not infringe privately owned rights. Reference herein to any specific commercial product, process, or service by trade name, trademark, manufacturer, or otherwise does not necessarily constitute or imply its endorsement, recommendation, or favoring by the United States Government or any agency thereof. The views and opinions of authors expressed herein do not necessarily state or reflect those of the United States Government or any agency thereof. 
water reflects the extent of water-rock interaction between the recharge water and the rock matrix. Tritium $\left({ }^{3} \mathrm{H}\right)$ and carbon- $14\left({ }^{14} \mathrm{C}\right)$ ages provide insight as to the nature of flow mechanisms and residence times of the unsaturated-zone water. Carbon $\left({ }^{14} \mathrm{C}\right)$ concentrations measured in the gaseous phase can provide information relevant to the residence time of carbon dioxide $\left(\mathrm{CO}_{2}\right)$ in this unusually thick unsaturated zone, and carbon isotopic data $\left(\delta^{13} \mathrm{C}\right)$ can help to identify carbon sources, a necessary step in ${ }^{14} \mathrm{C}$ age estimation. Carbon isotopic data of $\mathrm{CO}_{2}$ as a function of depth provide information on gas-flow mechanisms through the unsaturated zone as well as interactions with water and secondary calcite. Stable-isotopic compositions of hydrogen and oxygen in fracture and matrix waters can help to identify fracture/matrix interactions and sources of water. From the present chemical and isotopic data, along with previously published data collected at Yucca Mountain (for example, Yang and others, 1993 and 1996), conceptual hydrologic flow at Yucca Mountain can be described. Such investigations are important because ground water is the most likely transport medium for radionuclides from a repository to the accessible environment.

The purpose of this study is to obtain hydrochemical data, such as chemical compositions of pore and perched waters, and to determine flow mechanisms and residence times of gas and water and extent of fracture/matrix interactions for water. All of these can be used to calibrate flow and transport models, which can be used to estimate repository performance and safety.

A method of pore-water extraction for chemical-composition analysis developed by Yang and others (1988) and later modified by Mower and others $(1991,1994)$ was used in this study. Methods of porewater extraction for stable-isotope analysis were investigated in this study. Lithologic units that contain different minerals require different methods of porewater extraction for stable-isotope analyses of hydrogen and oxygen, such as compression extraction or vacuum distillation. Validations of the methods that were applicable to each lithologic unit are presented in the section on the stable-isotope compositions of pore water.

Yucca Mountain, located near the western boundary of the Nevada Test Site in southern Nye County about $160 \mathrm{~km}$ northwest of Las Vegas, consists of a rugged series of north-trending fault-block ridges that are composed of volcanic rocks that generally have an eastward tilt of $5^{\circ}$ to $10^{\circ}$ (Scott and Bonk, 1984). A thick section of volcanic rocks of Tertiary age overlies sedimentary strata of Paleozoic age in the Yucca Mountain region (Byers and others, 1976), with units of the 12.8 to 12.7 million-year-old (Ma) Paintbrush Group (Tertiary age) (Sawyer and others, 1994) forming most exposures (Christiansen and Lipman, 1965; Scott and Bonk, 1984). Two voluminous, densely welded ash-flow tuffs underlie Yucca Mountain (the Tiva Canyon and Topopah Spring Tuffs), separated by a much thinner interval of mostly nonwelded pyroclastic rocks. Stratigraphic nomenclature was recently revised and improved with many new isotopic ages (Sawyer and others, 1994) and that stratigraphic nomenclature is used here (fig. 1).

Numerous washes, generally underlain by alluvium, dissect the eastern side of the mountain. Nearly threefourths of the annual precipitation falls from October through April (Quiring, 1983). No perennial streams exist in the Yucca Mountain area. Surface runoff is infrequent and of short duration, occurring only as a direct result of intense precipitation or rapid snowmelt. Numerous geologic and hydrologic investigations conducted in the extensive characterization effort at Yucca Mountain have resulted in overlapping nomenclature to describe aspects of the geologic setting. Obvious differences in the hydrologic character of the welded and nonwelded sequences led Montazer and Wilson (1984) and Ortiz and others (1985) to develop thermo-mechanical (or hydrogeologic) designations for these units (fig. 1). Because the hydrogeologic units are based on similarity of properties (see Luckey and others, 1996, p. 17-20 for discussion), they do not correspond exactly with the stratigraphic units, in which physical properties (welding, degree of alteration, fracturing, permeability) vary significantly both laterally and vertically within single flow units. Note that both nomenclatures will be used in this report, but they are not interchangeable. For example, the Calico Hills nonwelded hydrogeologic unit (CHn) contains basal parts of the Topopah Spring Tuff, the entire Calico Hills Formation and the Prow Pass Tuff, and upper parts of the Bullfrog Tuff. Stratigraphic and hydrogeologic correlations are shown graphically in figure 1 .

This report describes ongoing investigations of unsaturated-zone water chemistry and isotope hydrology at Yucca Mountain. New data collected in 1996 include $\mathrm{CO}_{2}$ concentrations and carbon isotopic compositions $\left({ }^{13} \mathrm{C}\right.$ and ${ }^{14} \mathrm{C}$ ) of rock gas from several 


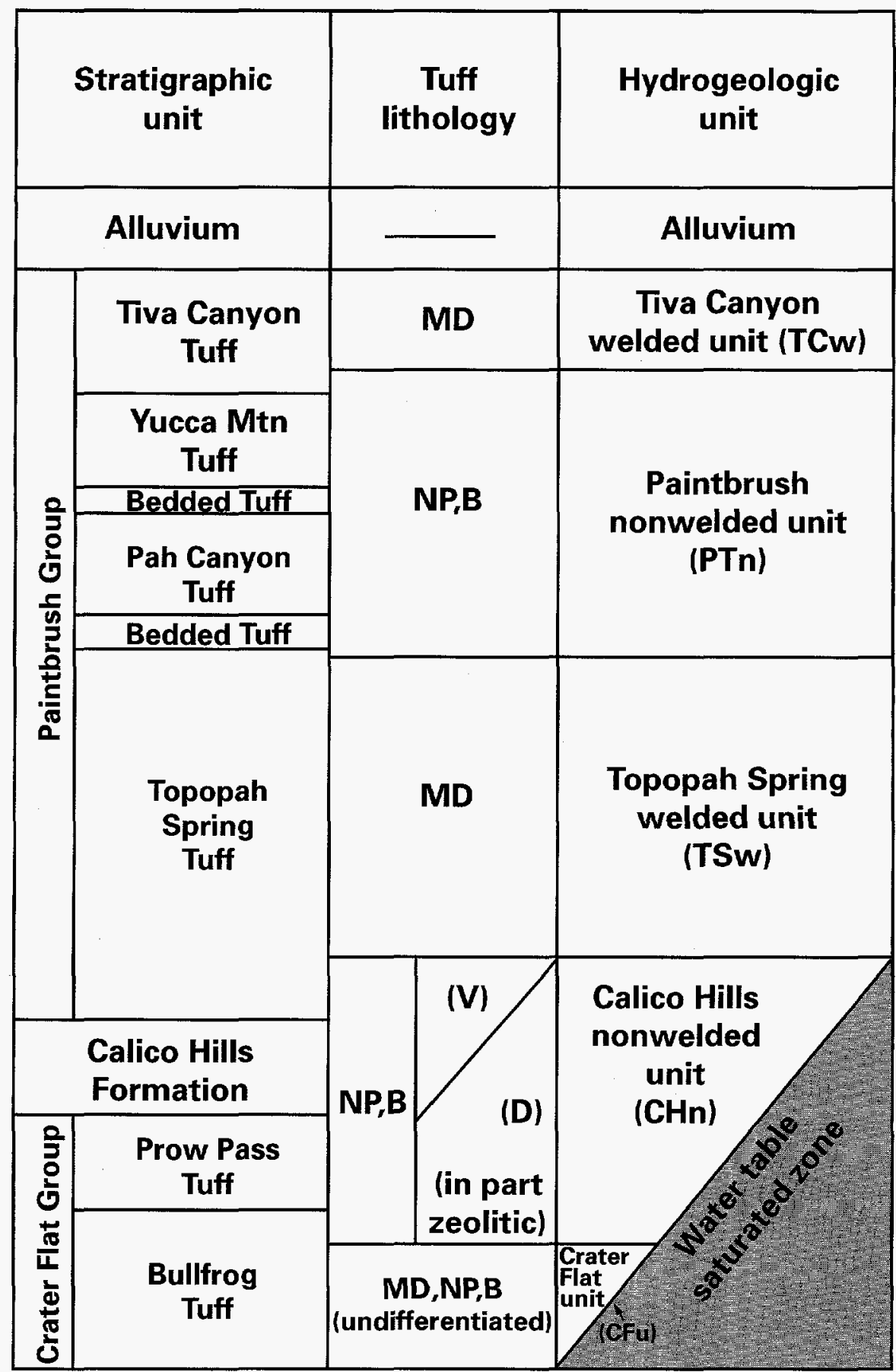

Figure 1. Hydrogeologic units at Yucca Mountain, Nevada [modified from Montazer and Wilson (1984), table 1]. MD, moderately to densely welded; NP, nonwelded to partially welded; B, bedded; (V), vitric; (D), devitrified. 
boreholes and major-ion concentrations and isotopic compositions of water from those and additional boreholes. Pore-water chemical compositions and ${ }^{14} \mathrm{C}$ data are from bedded tuff and the Calico Hills nonwelded unit (fig. 1). Limited ${ }^{3} \mathrm{H}$ data were obtained from intervals of each of the boreholes.

This investigation was conducted by the U.S. Geological Survey (USGS) in cooperation with the U.S. Department of Energy under Interagency Agreement DE-AI08-97NV12033.

\section{CHEMICAL AND RADIOACTIVE-ISOTOPE COMPOSITIONS $\left({ }^{3} \mathrm{H}\right.$ AND $\left.{ }^{14} \mathrm{C}\right)$ OF GAS AND PORE WATER FROM THE UNSATURATED ZONE}

A vacuum-reverse air-circulation drilling method (Whitfield, 1985) was used to drill all boreholes in the UZ drilling program. This method is intended to prevent contamination of the unsaturated rocks with drilling fluids and thus allow collection of native pore water and gas from the UZ for hydrochemical analysis. Locations of boreholes where gas and pore-water samples were collected are shown in figure 2. Borehole designation, drilling date, depth, and perched-water detections are given in table 1 . Abbreviated borehole names will be used throughout the text.

\section{Boreholes From Which Data Were Collected}

Drilling of geologic borehole NRG-6 was started on November 23,1992, and the borehole was cored to a depth of $335.3 \mathrm{~m}$, terminating in the Topopah Spring Tuff by March 3, 1993. NRG-7a was drilled beginning October 21, 1993, and cored to a depth of $461.3 \mathrm{~m}$ in the upper Calico Hills Formation by May 6, 1994. Cores above the Topopah Spring Tuff from these two boreholes were used for water extraction. Drilling of borehole SD-7 in the systematic drilling program was started on October 3, 1994; SD-7 was cored to $615.8 \mathrm{~m}$ with a $10-\mathrm{cm}$ bit by September 14,1995 , and completed to a depth of $815.3 \mathrm{~m}$ in the Tram Tuff on November 9, 1995. The water-table level in this borehole was at $633.3 \mathrm{~m}$ on November 13 , 1995. Perched water was reached in this borehole between $479.7 \mathrm{~m}$ and $488.3 \mathrm{~m}$. SD-9 was started on May 10, 1994, and completed to a depth of $677.6 \mathrm{~m}$, terminating in the Prow Pass Tuff on September 26, 1994. Perched water also was reached in this borehole at $453.8 \mathrm{~m}$. Borehole SD-12 drilling was started on January 23, 1994, and was completed to a depth of $660.3 \mathrm{~m}$, terminating in the Prow Pass Tuff on September 14,1994 . The static water table in SD-12 was located between $610.8 \mathrm{~m}$ and $612.9 \mathrm{~m}$. These SD holes yielded cores from both the Paintbrush nonwelded (PTn) and the Calico Hills nonwelded (CHn) hydrogeologic units for water extraction.

The UZ-14 borehole is located in Drill Hole Wash, $26.2 \mathrm{~m}$ from UZ-1. Drilling of the borehole was started on April 15,1993, and it was cored to a depth of $672.6 \mathrm{~m}$ by April 29, 1994, and completed to a total depth of $677.8 \mathrm{~m}$ on May 6,1994 . Chemical and radioactive-isotope data from UZ-14 have been previously reported from the interval from the surface to $528.8 \mathrm{~m}$ (Yang and others, 1996). This report includes data from the depth interval of 528.8 to $640.1 \mathrm{~m}$.

Gas samples were collected from the full depth range of SD-12 from 16 sampling ports. Boreholes SD-7 and SD-9 were not instrumented. Only one sample was collected from the Calico Hills nonwelded unit in SD-7, and two samples were obtained from SD-9 (one above and one below the Topopah Spring Tuff). For gas sampling from these open boreholes, an inflatable borehole liner was used.

\section{Sample Collection and Analysis}

Details of drilling were designed to enhance analysis. After a borehole was drilled, the atmospheric air that entered during the drilling process was removed by evacuation from the surface with a largecapacity pump. All drilling air was tagged with a tracer: sulfur hexafluoride $\left(\mathrm{SF}_{6}\right)$. The tracer concentration in the evacuated air was continuously monitored, and disappearance of the $\mathrm{SF}_{6}$ [concentration less than 0.1 part per million $(\mathrm{ppm})]$ assured essentially complete removal of the drilling air. Packers were installed inside the open boreholes in SD-7 and SD-9 for gas-sample collections. The diameter of the rotary core was $6 \mathrm{~cm}$. Cores from the rock formation were obtained using a $1.5-\mathrm{m}$ core barrel with a split inner tube. Core recovery typically was greater than 90 percent. Three pieces of continuous, unfragmented solid core, each approximately $15 \mathrm{~cm}$ long, are needed to obtain enough water for age and chemical determinations. 


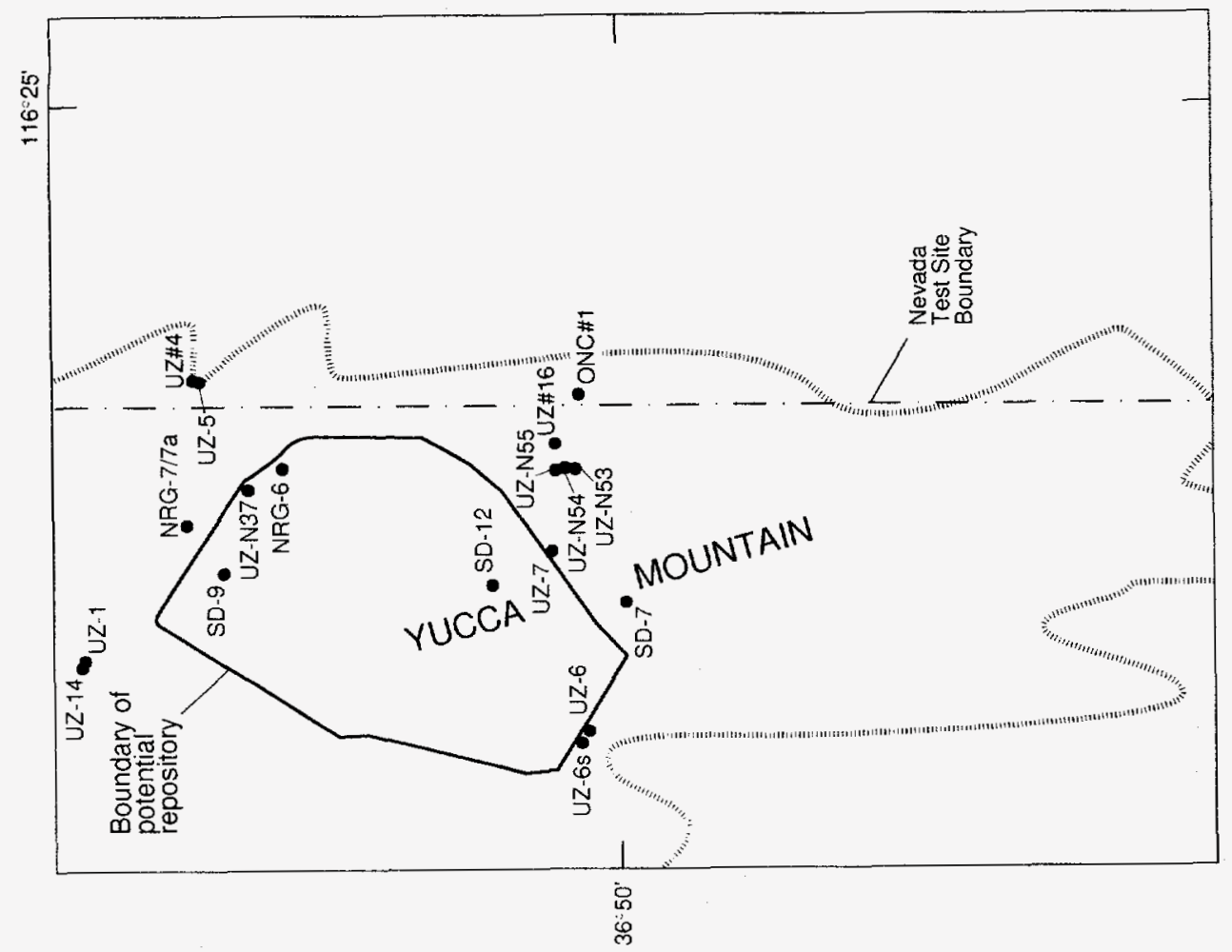

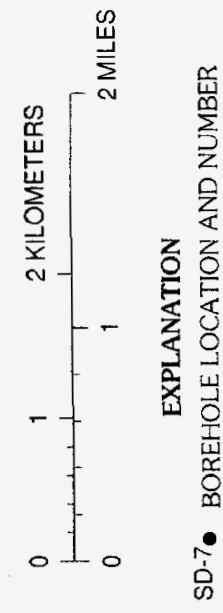

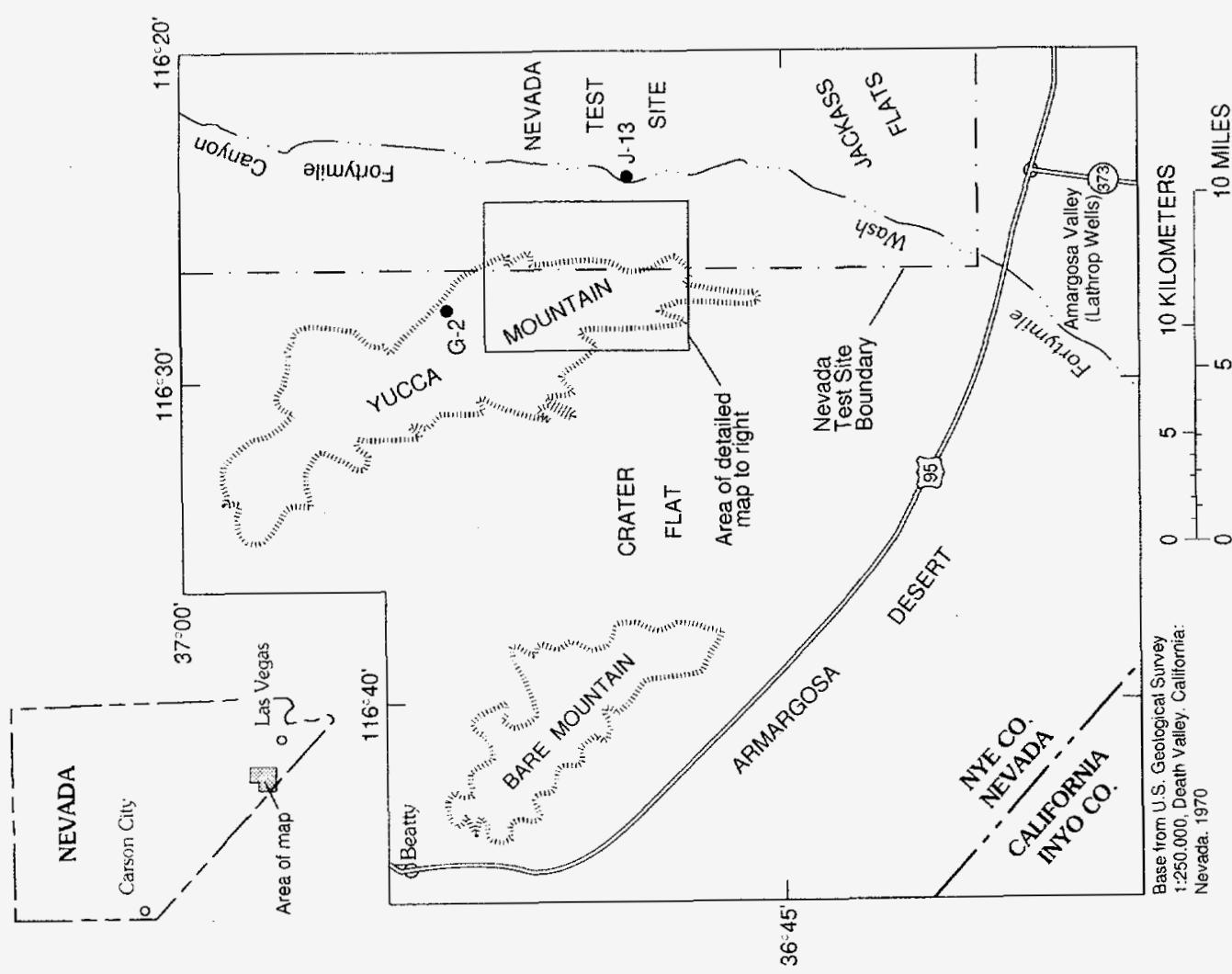

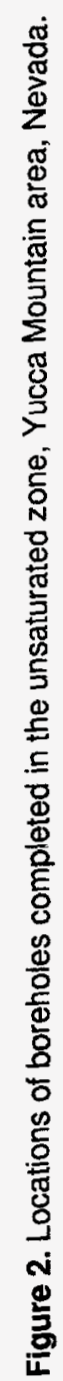


Table 1. Borehole designation, drilling date, depth, and perched-water occurrence of selected boreholes at Yucca Mountain area

[m, meter; ---, not found (source of information: Yang and others, 1996; Rautman and Engstrom, 1996)]

\begin{tabular}{lccccc}
\hline $\begin{array}{c}\text { Borehole } \\
\text { designation }\end{array}$ & $\begin{array}{c}\text { Borehole } \\
\text { abbreviation }\end{array}$ & Start & Finish & $\begin{array}{c}\text { Depth of } \\
\text { borehole } \\
(\mathbf{m})\end{array}$ & $\begin{array}{c}\text { Perched water } \\
\text { detected at depth } \\
(\mathbf{m})\end{array}$ \\
\hline USW NRG-6 & NRG-6 & $11-23-92$ & $03-03-93$ & 335.3 & -- \\
USW NRG-7a & NRG-7a & $10-21-93$ & $05-06-94$ & 461.3 & 460.2 \\
USW SD-7 & SD-7 & $10-03-94$ & $11-09-95$ & 815.3 & 479.7 \\
USW SD-9 & SD-9 & $05-10-94$ & $09-26-94$ & 677.6 & 453.8 \\
USW SD-12 & SD-12 & $01-23-94$ & $09-14-94$ & 660.3 & -- \\
USW UZ-14 & UZ-14 & $04-15-93$ & $05-06-94$ & 677.8 & 384.6 \\
\hline
\end{tabular}

Sample-handling procedures affect the success of extracting uncontaminated water from cores of tuff. Evaporation of pore water increases with sample exposure time in a dry climate, and strict precautions were taken to avoid loss of moisture or contamination of pore water in the cores (Striffler and Peters, 1993). Each core was wrapped in a thin plastic sheet, placed inside a Lexan liner, and capped on both ends of the Lexan liner. The Lexan-contained core was then placed inside a thick aluminum-foil bag and heatsealed for moisture protection. The sealed cores were packed carefully into plastic coolers and transported from the drill site to the Sample Management Facility (SMF) at Jackass Flats, Nevada, for log-in and storage. The storage temperature was kept at $6^{\circ}$ to $9^{\circ} \mathrm{C}$ until the cores were removed for pore-water extraction.

Analyses were contracted out by the USGS except for tritium, gas composition, and $\mathrm{CO}_{2}$ concentration, which were analyzed by the USGS in Denver, Colorado.

\section{Collection of Gas Samples from Boreholes SD-7, SD-9, and SD-12}

The whole-gas (WG) method or batch sampling was used to collect samples for $\delta^{13} \mathrm{C}$ analysis. A gas stream was allowed to flow into a Mylar-balloon sample container to purge the balloon several times before sampling. When the gas had filled up the balloon, the sample container was removed from the flow line. The collection time was less than 5 minutes.

The molecular-sieve (MS) method was used to collect gas samples for ${ }^{14} \mathrm{C}$ and $\delta^{13} \mathrm{C}$ analyses. The gas stream was initially passed continuously through a silica-gel tower to remove moisture, then through a 300-mL stainless-steel cylinder containing a 50-nanometer (50-nm), anhydrous (dehydrated under a vacuum at $350^{\circ} \mathrm{C}$ ) MS to trap the $\mathrm{CO}_{2}$ gas. The $\mathrm{CO}_{2}$ gas trapped in the MS was degassed in the laboratory to release the trapped $\mathrm{CO}_{2}$. Released $\mathrm{CO}_{2}$ was collected in a cold trap, the volume of $\mathrm{CO}_{2}$ was measured, and the $\mathrm{CO}_{2}$ was transferred to storage cylinders.

Boreholes SD-7 and SD-9 were open boreholes. For gas sampling from these boreholes, an inflatable borehole liner was used. Gas samples were collected from individual tubes connected to open ports located along the length of an impermeable membrane liner. The liner is inflated to seal the formation wallrock from direct contact with the atmosphere. Two intervals were packed in SD-9: one from the surface to $453.5 \mathrm{~m}$ (lithologic units of Tiva Canyon and Topopah Spring Tuff), and the other from 453.7 to $560 \mathrm{~m}$ (Calico Hills nonwelded hydrogeologic unit). Only one interval was packed in SD-7, from 508 to $633 \mathrm{~m}$ (Calico Hills nonwelded unit). Gas data from SD-12 were obtained from the entire borehole, in contrast to the SD-7 and SD-9 data obtained from selected intervals.

\section{Collection of Pore-Water Samples from SD-7, SD-9, SD-12, NRG-6, and NRG-7a}

Pore water was extracted from the cores by using a high-pressure, one-dimensional (uniaxial) compression procedure (Mower and others, 1991, 1994). The compression operations were described in 
a previous report (Yang and others, 1996). A pressure of 103.4 megapascals (MPa) was initially applied and gradually increased to a final pressure of $827 \mathrm{MPa}$. Additional pore water was extracted by injecting dry nitrogen gas (greater than 99.99 percent pure) into the pore space and by forcing out pore water at the end of compression. The extracted water was filtered through 0.45 -micrometer $(0.45-\mu \mathrm{m})$ Nuclepore filters into suitable bottles. Samples for major ions were stored in polyethylene bottles, and samples for stable isotopes and ${ }^{14} \mathrm{C}$ were stored in glass bottles.

\section{Analyses of Gas and Water Samples}

Gas samples for ${ }^{14} \mathrm{C}$ and $\delta^{13} \mathrm{C}$ analyses were sent to the Desert Research Institute (DRI) in Las Vegas, Nevada, for conventional liquid scintillation counting for ${ }^{14} \mathrm{C}$ and mass spectrometry for $\delta^{13} \mathrm{C}$. The precision of $\delta^{13} \mathrm{C}$ analysis was about 0.2 per mil [one standard deviation $(1 \sigma)$ ]. Uncertainty in the ${ }^{14} \mathrm{C}$ measurement was 0.7 percent modern carbon (pmc) $(1 \sigma)$.

Cation concentrations were measured using inductively coupled plasma emission spectroscopy, and anion concentrations were measured using ion chromatography at Huffman Laboratories, Inc., in Golden, Colorado. The analytical error is \pm 5 percent for all major ions except sulfate, for which the error is \pm 10 percent. Charge balance is calculated by subtracting total milliequivalent anions from total milliequivalent cations divided by the total milliequivalents of cation and anion multiplied by 100 . However, silica concentration is not considered in the charge balance because of difficulty in assessing charge on silicate species. The tritium was analyzed at the USGS Yucca Mountain Project Branch (YMPB) UZ Hydrochemistry Laboratory using procedures published by Thatcher and others (1977). Tritium values were calculated by regressing to the date of sample collection so that tritium decay is considered between sample collection and date of analysis. Tritium errors were approximately 4 tritium units $(1 \sigma)$ [ 1 tritium unit $(1 \mathrm{TU})=1{ }^{3} \mathrm{H}$ atom per $10^{18}{ }^{1} \mathrm{H}$ atoms]. Carbon- 14 and $\delta^{13} \mathrm{C}$ samples were sent to Beta Analytic, Inc., in Miami, Florida, for analysis by Tandem Accelerator Mass Spectrometer (TAMS). Uncertainty in $\delta^{13} \mathrm{C}$ values was 0.2 per mil and for ${ }^{14} \mathrm{C}$ was 0.7 pmc. All uncertainties quoted are one standard deviation $(1 \sigma)$.
The aluminum (A1) concentration in pore water is mainly controlled by aluminum silicates. Except for extreme $\mathrm{pH}$ conditions, dissolved $\mathrm{Al}$ is usually below the detection limit (less than 1 milligram per liter $(\mathrm{mg} / \mathrm{L})$. Very fine filters $(0.1 \mu \mathrm{m})$ must be used to remove particulate $\mathrm{Al}$ to obtain trustworthy measured concentrations for dissolved Al. Because 0.45- $\mu \mathrm{m}$ filter paper was used for pore-water filtration in this study, reported $\mathrm{Al}$ values for pore waters are total $\mathrm{Al}$ concentration, including dissolved and particulate components.

\section{Data and Interpretations of Chemical and Radioactive-Isotope Analyses}

This section presents hydrochemical data obtained to date and interpretations of those data related to possible flow mechanisms and residence times in the UZ. Data are chemical compositions (major cations and anions) and isotopic concentrations, which include ${ }^{3} \mathrm{H},{ }^{14} \mathrm{C}$, and $\delta^{13} \mathrm{C}$ of pore water in cores obtained from boreholes in the UZ. Preliminary conceptual hydrologic flow models are derived from the present chemical and isotopic data, along with previously published data collected at Yucca Mountain (Yang and others, 1996).

\section{Spatial Variabilities in Chemical Compositions}

Recent analyses augment previously published data from major-ion chemical investigations. Majorion chemical compositions of pore water from UZ-14, from the surface to $528.7 \mathrm{~m}$ were published previously by Yang and others (1996). Additional data from waters obtained mostly below $528.7 \mathrm{~m}$ are presented in table 2 and plotted in figure 3. NRG-6 and NRG-7a major-ion chemical compositions are presented in table 3 and plotted in figure 4. Major-ion chemical compositions of samples from boreholes SD-7, SD-9, and SD- 12 are presented in table 4 and plotted in figure 5. Several samples from the Topopah Spring Tuff were analyzed because not many samples of pore water could be extracted for chemical analysis. In the previous report by Yang and others (1996), only a few samples from the caprock unit (usage after Scott and Bonk, 1984) of the Topopah Spring Tuff from UZ-4 and UZ-5 were analyzed. 
Table 2. Chemical composition of pore-water samples from borehole USW UZ-14, Yucca Mountain area

[- - -, data not available; 0 , values below detection limit; $\mathrm{m}$, meter; $\mu \mathrm{S} / \mathrm{cm}$, microsiemens per centimeter at 25 degrees Celsius; $\mathrm{mg} / \mathrm{L}$, milligrams per liter; charge balance, (meq cation - meq anion)/ (meq cation + meq anion) $\times 100$; PTn, Paintbrush nonwelded; CHn, Calico Hills nonwelded; PP, Prow Pass Tuff; YM, Yucca Mountain Tuff; CFu, Crater Flat unit]

\begin{tabular}{|c|c|c|c|c|c|c|c|c|c|c|c|c|c|c|c|c|c|}
\hline $\begin{array}{c}\text { Sample } \\
\text { identification }\end{array}$ & $\begin{array}{c}\text { Symbol } \\
\text { in Piper } \\
\text { diagram } \\
\text { (fig. 3) }\end{array}$ & $\begin{array}{l}\text { Average } \\
\text { depth } \\
\text { (m) }\end{array}$ & Date & pH & $\begin{array}{c}\text { Specific } \\
\text { conduct- } \\
\text { ance } \\
(\mu S / \mathrm{cm})\end{array}$ & $\begin{array}{c}\text { Calcium } \\
\text { Ca } \\
\text { (mg/L) }\end{array}$ & $\begin{array}{c}\text { Magne- } \\
\text { sium } \\
\mathrm{Mg} \\
(\mathrm{mg} / \mathrm{L})\end{array}$ & $\begin{array}{c}\text { Sodium } \\
\mathrm{Na} \\
(\mathrm{mg} / \mathrm{L})\end{array}$ & $\begin{array}{c}\text { Silica } \\
\mathrm{SiO}_{2} \\
(\mathrm{mg} / \mathrm{L})\end{array}$ & 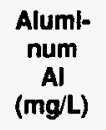 & $\begin{array}{c}\text { Bicar- } \\
\text { bonate } \\
\mathrm{HCO}_{3} \\
(\mathrm{mg} / \mathrm{L})\end{array}$ & $\begin{array}{c}\text { Car- } \\
\text { bonate } \\
\mathrm{CO}_{3} \\
(\mathrm{mg} / \mathrm{L})\end{array}$ & $\begin{array}{c}\text { Chlorine } \\
\text { Cl } \\
\text { (mg/L) }\end{array}$ & $\begin{array}{c}\text { Bromine } \\
\text { Br } \\
\text { (mg/L) }\end{array}$ & $\begin{array}{c}\text { Nitrate } \\
\mathrm{NO}_{3} \\
\text { (mg/L) }\end{array}$ & $\begin{array}{c}\text { Sulfate } \\
\text { SO }_{4} \\
(\mathrm{mg} / \mathrm{L})\end{array}$ & $\begin{array}{l}\text { Charge } \\
\text { balance }\end{array}$ \\
\hline $\begin{array}{l}\mathrm{UZ}-14 / 45.0- \\
45.4 / \mathrm{PTn} / \mathrm{YM}\end{array}$ & 1 & 13.78 & $09-19-94$ & 8.6 & 1,320 & 19.6 & 6.1 & 249.3 & 59.5 & 1 & 245 & 18 & 245 & 0 & 35 & 33 & -1.8 \\
\hline $\begin{array}{l}\text { UZ-14/1735.3- } \\
1735.53 / \mathrm{CHn}\end{array}$ & a & 528.95 & $05-04-95$ & 9.0 & 700 & 1.7 & 0.0 & 158 & 46.9 & 0.0 & 288 & 18 & 37.4 & 0.0 & 3.4 & 15.4 & 1.5 \\
\hline $\begin{array}{l}\mathrm{UZ}-14 / 1804.5- \\
1804.7 \mathrm{CHn} / \mathrm{PP}\end{array}$ & $\mathrm{b}$ & 550.04 & $05-10-95$ & 8.7 & 480 & 0.5 & 0.2 & 111 & 43.0 & 1.0 & 181 & 12 & 25.1 & 0.0 & 15.3 & 14.9 & 2.5 \\
\hline $\begin{array}{l}\text { UZ-14/1825.8- } \\
1826.0 / \mathrm{CHn} / \mathrm{PP}\end{array}$ & $\mathrm{c}$ & 556.53 & $06-22-95$ & 9.4 & 590 & 0.6 & 0.1 & 138 & 47.9 & 0.9 & 42 & 112 & 23.5 & 0.0 & 11.8 & 12.9 & 4.3 \\
\hline $\begin{array}{l}\text { UZ-14/1854.89- } \\
1855.1 / \mathrm{CHn} / \mathrm{PP}\end{array}$ & $d$ & 565.40 & $07-07-95$ & 9.2 & 460 & 0.5 & 0.2 & 107 & 34.5 & 1.6 & 181 & 23 & 18.8 & 0.0 & 13.7 & 15.9 & -1.4 \\
\hline $\begin{array}{l}\text { UZ-14/1865.7- } \\
\text { 1865.9/CHn/PP }\end{array}$ & $\mathrm{e}$ & 568.70 & $07-20-95$ & 9.1 & 480 & 0.3 & 0.0 & 115 & 30.4 & 0.8 & 204 & 18 & 17.3 & 0.0 & 11.7 & 12.6 & 1.4 \\
\hline $\begin{array}{l}\text { UZ-14/2014,7- } \\
2014.9 / \mathrm{CHn} / \mathrm{PP}\end{array}$ & f & 614.11 & $07-27-95$ & 9.3 & 1,520 & 3.2 & 0.2 & 392 & 37.7 & 0.2 & 409 & 219 & 32.1 & 0.0 & 1.8 & 22.2 & 5.6 \\
\hline $\begin{array}{l}\text { UZ-14/2015.2- } \\
2025.7 \mathrm{CHn} / \mathrm{PP}\end{array}$ & g & 615.85 & $08-02-95$ & 9.2 & 1,450 & 0.9 & 0.0 & 312.7 & 68.6 & - . & 339 & 176 & 23.2 & 0.1 & 0.5 & 21.7 & 4.2 \\
\hline $\begin{array}{l}\text { UZ-14/2025.1- } \\
2025.3 / \mathrm{CHn} / \mathrm{PP}\end{array}$ & $\mathrm{h}$ & 617.28 & 08-02-95 & 9.3 & 1,550 & 4.0 & 0.2 & 414 & 54.8 & 0.2 & 493 & 189 & 36.5 & 0.0 & 6.9 & 26.3 & 6.3 \\
\hline $\begin{array}{r}\text { UZ-14/2095.6- } \\
2095.8 / \mathrm{CFu}\end{array}$ & $\mathrm{n}$ & 638.71 & $08-18-95$ & 9.0 & 530 & 0.0 & 0.0 & 112.8 & 63.0 & - - & 143 & 29 & 30.0 & 0.1 & 0.3 & 29.0 & 1.3 \\
\hline $\begin{array}{c}\text { UZ-14/2104.05/ } \\
\text { bottom/CFu }\end{array}$ & $*$ & 641.33 & $04-22-94$ & 9.4 & $\cdots$ & 2.1 & 0.7 & 143 & 352 & 38 & 229 & 19 & 10 & 0 & 4 & 23 & 10.1 \\
\hline
\end{tabular}




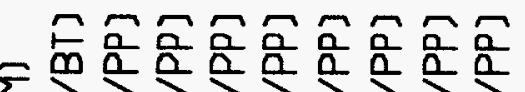

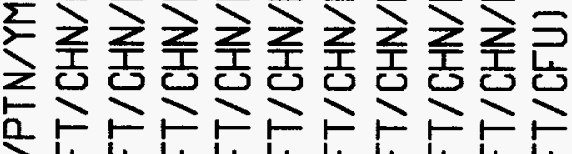

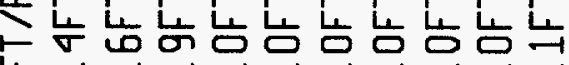

录 ஸ

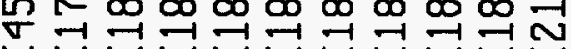

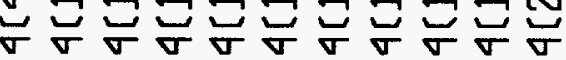

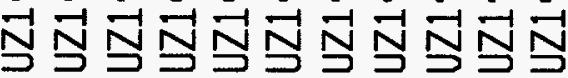

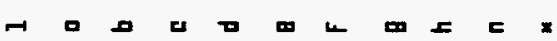
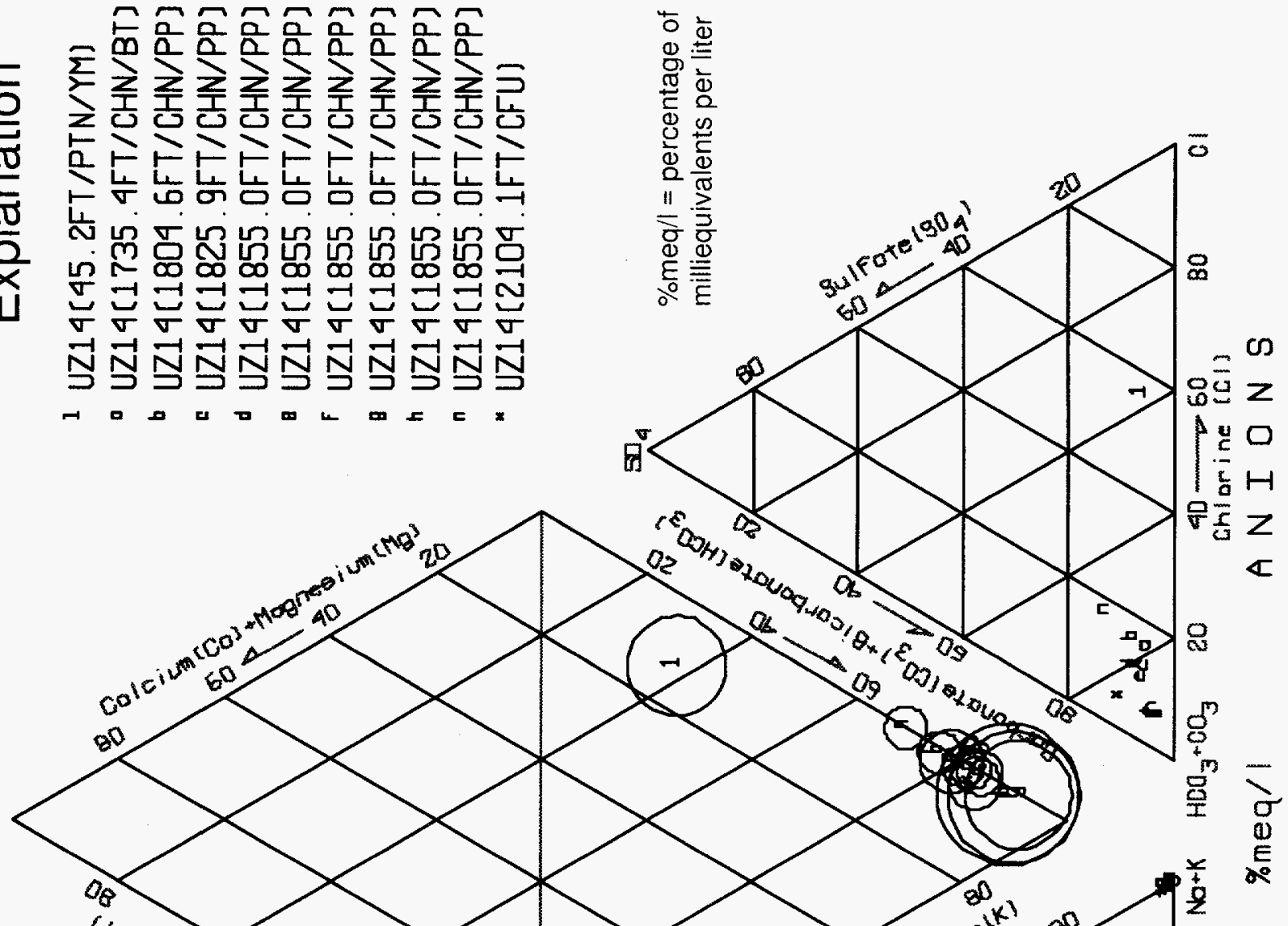

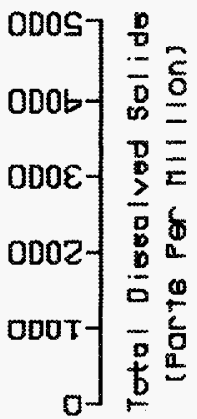

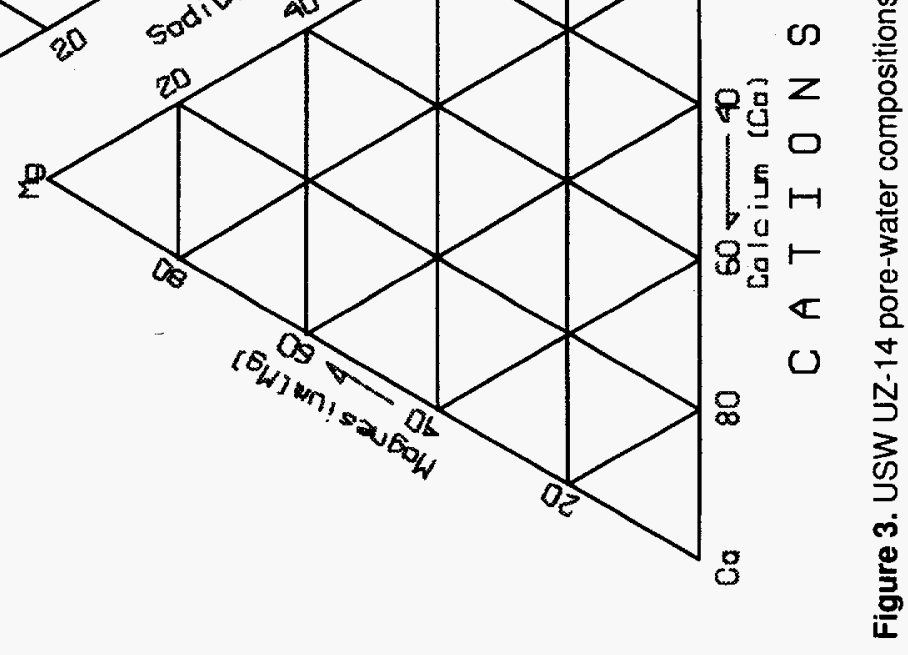


Table 3. Chemical composition of pore-water samples from boreholes USW NRG-6 and NRG-7a, Yucca Mountain area

[m, meter; $\mu \mathrm{S} / \mathrm{cm}$, microsiemens per centimeter at 25 degrees Celsius; $\mathrm{mg} / \mathrm{L}$, milligrams per liter; 0 , values below detection limit; charge balance, (meq cation - meq anion)/

(meq cation + meq anion); PTn, Paintbrush nonwelded; BT, bedded tuff; PC, Pah Canyon; CHn, Calico Hills nonwelded]

\begin{tabular}{|c|c|c|c|c|c|c|c|c|c|c|c|c|c|c|c|c|c|}
\hline $\begin{array}{c}\text { Sample } \\
\text { identification }\end{array}$ & $\begin{array}{l}\text { Symbol } \\
\text { In Piper } \\
\text { diagram } \\
\text { (fig. 4) }\end{array}$ & $\begin{array}{l}\text { Average } \\
\text { depth } \\
\text { (m) }\end{array}$ & Date & pH & $\begin{array}{c}\text { Specific } \\
\text { conduct- } \\
\text { ance } \\
(\mu \mathrm{S} / \mathrm{cm})\end{array}$ & $\begin{array}{c}\text { Calcium } \\
\text { Ca } \\
\text { (mg/L) }\end{array}$ & $\begin{array}{c}\text { Magne- } \\
\text { slum } \\
\mathrm{Mg} \\
(\mathrm{mg} / \mathrm{L})\end{array}$ & $\begin{array}{c}\text { Sodium } \\
\mathrm{Na} \\
(\mathrm{mg} / \mathrm{L})\end{array}$ & $\begin{array}{c}\text { Silica } \\
\mathrm{SiO}_{2} \\
\text { (mg/L) }\end{array}$ & $\begin{array}{c}\underset{\text { Alumi- }}{\text { num }} \\
\text { Al' }^{1} \\
(\mathbf{m g} / \mathrm{L})\end{array}$ & $\begin{array}{c}\text { Bicar- } \\
\text { bonate } \\
\mathrm{HCO}_{3} \\
(\mathrm{mg} / \mathrm{L})\end{array}$ & $\begin{array}{c}\text { Car- } \\
\text { bonate } \\
\mathrm{CO}_{3} \\
(\mathrm{mg} / \mathrm{L})\end{array}$ & $\begin{array}{c}\text { Chlorine } \\
\text { Cl } \\
\text { (mg/L) }\end{array}$ & $\begin{array}{c}\text { Bromine } \\
\mathbf{B r} \\
(\mathbf{m g} / \mathrm{L})\end{array}$ & $\begin{array}{c}\text { Nitrate } \\
\mathrm{NO}_{3} \\
\text { (mg/L) }\end{array}$ & $\begin{array}{c}\text { Sulfate } \\
\text { SO }_{4} \\
\text { (mg/L) }\end{array}$ & $\begin{array}{l}\text { Charge } \\
\text { balance }\end{array}$ \\
\hline NRG-6/158.2 158.6/PTn/BT & A & 48.28 & $07-06-94$ & 6.8 & 1,070 & 122 & 23.3 & 35.6 & 97.4 & 0 & 34 & 0 & 185 & 1 & 32 & 159 & -0.2 \\
\hline NRG-6/160.8-161.2/PTn/BT & B & 49.07 & $01-17-95$ & 8.0 & 860 & 104 & 18 & 35.0 & 84.0 & 1.6 & 55 & 0 & 148 & 0.9 & 35 & 139 & -2.0 \\
\hline NRG-6/171.0-171.3/PTn/BT & $\mathrm{C}$ & 52.18 & $07-21-94$ & 7.4 & 620 & 70.5 & 11.5 & 29.2 & 79.4 & 0 & 48 & 13 & 58 & 0 & 43 & 94 & 2.0 \\
\hline NRG-6/175.6-176.0/PTn/PC & $\mathrm{D}$ & 53.58 & $08-01-94$ & 7.3 & 520 & 49.2 & 8.6 & 29.4 & 78.1 & 0 & 60 & 0 & 47 & 0 & 42 & 64 & 1.5 \\
\hline NRG-6/219.9-220.2/PTn/PC & $\mathrm{E}$ & 67.09 & $11-30-94$ & 7.4 & 660 & 24.3 & 4.2 & 99.3 & 61.4 & 1 & 92 & 0 & 77 & 0 & 47 & 77 & -1.3 \\
\hline NRG-6/244.6-245.0/PTn & $\mathrm{F}$ & 74.62 & $01-10-95$ & 7.2 & 630 & 33 & 4.9 & 72.0 & 51.0 & 0.6 & 61 & 0 & 49 & 0.1 & 40 & 115 & -2.3 \\
\hline NRG-6/255.9-256.1/PTn & G & 78.03 & $01-12-95$ & 6.7 & 1,920 & 176 & 19 & 215.0 & 68.0 & 0.9 & 61 & 0 & 115 & 0.0 & 35 & 840 & -6.2 \\
\hline NRG-7A/165.8-166.0/PTn/BT & $\mathrm{H}$ & 50.57 & 09-08-95 & 7.3 & 500 & 55.3 & 5.6 & 31.7 & 68.3 & 0 & 89.0 & 0 & 38.9 & 1.3 & 45.5 & 63.4 & 0 \\
\hline NRG-7A/258.0-258.4/PTn/BT & I & 78.70 & $10-17-95$ & 8.3 & 595 & 43 & 3.7 & 82 & 68.9 & 1.0 & 128 & 0 & 53.6 & 0.3 & 43.7 & 65.5 & 2.9 \\
\hline NRG-7A/1483.5-1483.8/CHn/BT & $\mathbf{J}$ & 452.22 & $10-25-95$ & 8.0 & 580 & 61 & 0.6 & 94 & 48.6 & 0.3 & 323 & 0 & 33.1 & 0.1 & 16.7 & 24.4 & 1.3 \\
\hline NRG-7A/1492.7-1493.1/CHn/BT & $\mathrm{K}$ & 455.04 & $05-26-94$ & 8.6 & 510 & 30.6 & 0.3 & 74.7 & 71.5 & 0.0 & 104 & 34 & 39 & 0 & 18 & 23 & 0.9 \\
\hline NRG-7A/1498.6-1498.9/CHn/BT & $\mathrm{L}$ & 456.83 & $06-28-94$ & 8.3 & 500 & 28.7 & 0.5 & 73.2 & 83.0 & 1 & 156 & 0 & 50 & 0 & 17 & 18 & 0.4 \\
\hline
\end{tabular}

${ }^{1} 0.45-\mu \mathrm{m}$ filter paper was used; hence, particulate aluminum is likely included in these values. 


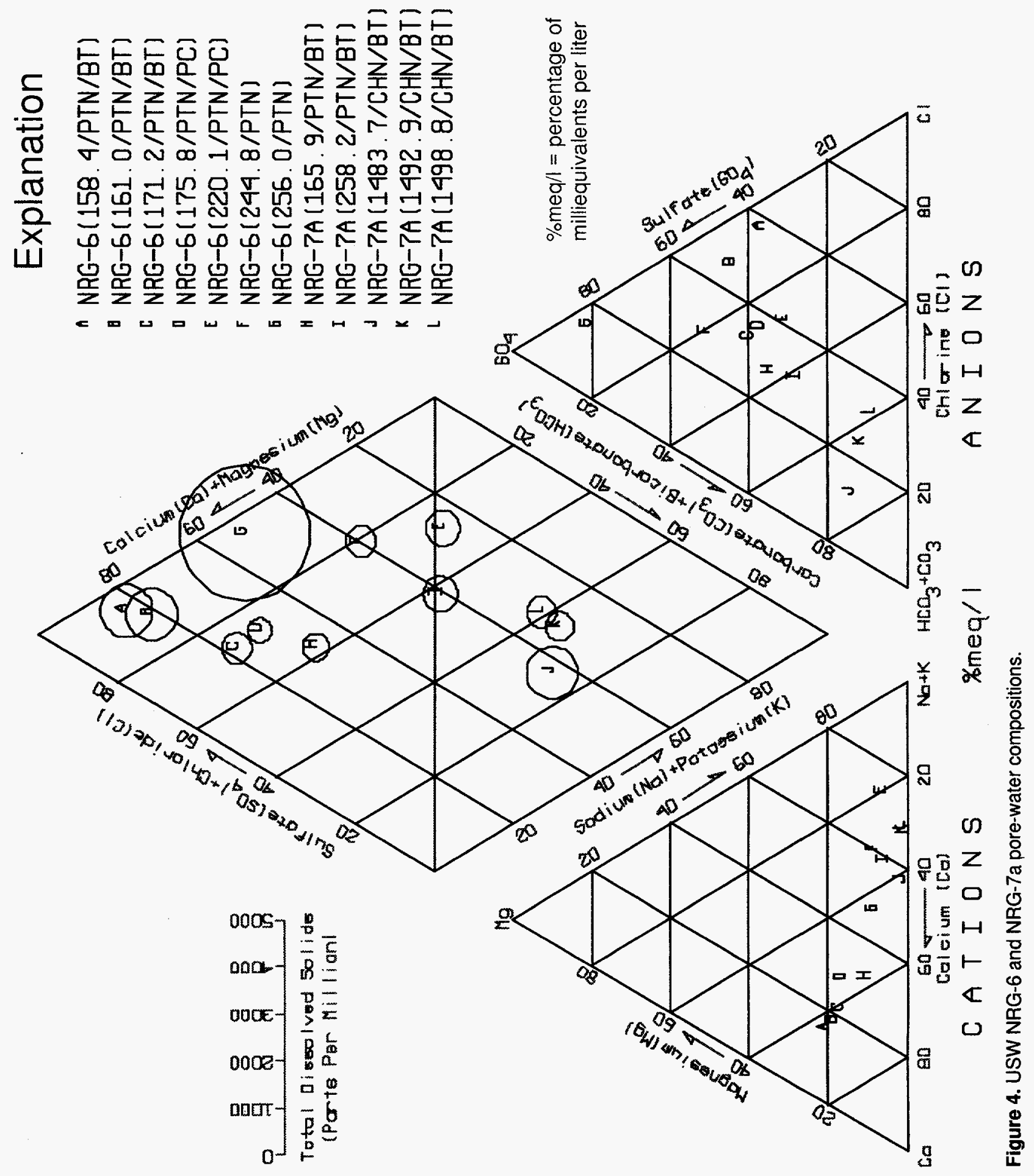


Table 4. Chemical composition of pore-water samples from boreholes USW SD-7, SD-9, and SD-12, Yucca Mountain area

[m, meter; $\mu \mathrm{S} / \mathrm{cm}$, microsiemens per centimeter at 25 degrees Celsius; $\mathrm{mg} / \mathrm{L}$, milligrams per liter; ---, data not available; 0 , values below detection limit; -, less than; N/S, not sampled; charge balance, (meq cation - meq anion)/(meq cation + meq anion) $\times 100$; PTn, Paintbrush nonwelded; CHn, Calico Hills nonwelded; PP, Prow Pass Tuff; BT, bedded tuff; TSw, Topopah Spring Tuff; YM, Yucca Mountain Tuff]

\begin{tabular}{|c|c|c|c|c|c|c|c|c|c|c|c|c|c|c|c|c|c|}
\hline $\begin{array}{c}\text { Sample } \\
\text { identification }\end{array}$ & $\begin{array}{c}\text { Symbol } \\
\text { in Piper } \\
\text { diagram } \\
\text { fig. } 5\end{array}$ & $\begin{array}{l}\text { Average } \\
\text { depth } \\
\text { (m) }\end{array}$ & Date & pH & $\begin{array}{c}\text { Specific } \\
\text { conduc- } \\
\text { tance } \\
(\mu \mathrm{S} / \mathrm{cm})\end{array}$ & $\begin{array}{c}\text { Calcium } \\
\text { Ca } \\
\text { (mg/L) }\end{array}$ & $\begin{array}{c}\text { Magne-- } \\
\text { sium } \\
\mathrm{Mg} \\
(\mathrm{mg} / \mathrm{L})\end{array}$ & $\begin{array}{c}\text { Sodium } \\
\mathrm{Na} \\
(\mathrm{mg} / \mathrm{L})\end{array}$ & $\begin{array}{c}\text { Sllica } \\
\mathrm{SiO}_{2} \\
(\mathrm{mg} / \mathrm{L})\end{array}$ & $\begin{array}{c}\text { Potas- } \\
\text { sium } \\
\text { K } \\
(\mathrm{mg} / \mathrm{L})\end{array}$ & $\begin{array}{l}\text { Bicar- } \\
\text { bonate } \\
\mathrm{HCO}_{3} \\
(\mathrm{mg} / \mathrm{L})\end{array}$ & $\begin{array}{c}\text { Car- } \\
\text { bonate } \\
\mathrm{CO}_{3} \\
\text { (mg/L) }\end{array}$ & $\begin{array}{c}\text { Chlo- } \\
\text { rine } \\
\mathrm{Cl} \\
(\mathbf{m g} / \mathrm{L})\end{array}$ & $\begin{array}{c}\text { Bromine } \\
\text { Br } \\
(\mathbf{m g} / \mathrm{L})\end{array}$ & $\begin{array}{c}\text { Nitrate } \\
\mathrm{NO}_{3} \\
\text { (mg/L) }\end{array}$ & $\begin{array}{c}\text { Sulfate } \\
\mathrm{SO}_{4} \\
(\mathrm{mg} / \mathrm{L})\end{array}$ & $\begin{array}{l}\text { Charge } \\
\text { balance }\end{array}$ \\
\hline SD-7/339.7-340.2/PTn/BT & a & 103.63 & $04-25-96$ & 7.2 & 720 & 110 & 15 & 20 & 82.8 & --- & 220 & 0 & 77.2 & 0.5 & 1.5 & 76.7 & 1.3 \\
\hline SD-7/370.3-370.6/TSnw & $\mathrm{b}$ & 112.93 & $05-14-97$ & 6.7 & 1,420 & 289 & 0.2 & 39 & 25.9 & -- & 73 & 0 & 133 & 0.4 & 0.6 & 650 & -6.7 \\
\hline SD-7/1498.4-1498.6/CHn & c & 456.74 & $04-05-96$ & 7.4 & 405 & 31 & 0.6 & 67 & 63.5 & --- & 171 & 0 & 28.9 & 0.4 & 13.6 & 14.1 & 4.4 \\
\hline SD-7/1524.6-1525.7/CHn & d & 464.85 & $01-12-96$ & 7.7 & 490 & 38.7 & 0.9 & 57.6 & 68.6 & 7 & 203 & 0 & 30.1 & 0.1 & 12.4 & 15.9 & -0.2 \\
\hline SD-7/1558.4-1558.6/CHn & $\mathrm{e}$ & 475.03 & $03-13-96$ & 7.4 & 370 & 39 & 0.9 & 43 & 69.3 & -- & 171 & 0 & 25.1 & 0.1 & 9.0 & 14.9 & -1.0 \\
\hline SD-7/1600.1-1600.3/CHn & $\mathrm{f}$ & 487.74 & $07-14-97$ & 7.6 & 380 & 23 & 0.6 & 59 & 66.7 & $\cdots$ & 150 & 0 & 31 & 0.0 & 5.7 & 12 & 1.2 \\
\hline SD-7/1617.0-1617.2/CHn & g & 492.87 & $10-25-96$ & 7.2 & 570 & 40.2 & 0.4 & 79 & 62.2 & --- & 194 & 0 & 65 & 0.1 & 11.0 & 14 & 0.0 \\
\hline SD-7/1890.9/CHn/PP & $\mathrm{h}$ & 576.35 & $05-09-97$ & 8.7 & 850 & 0 & 0.2 & 206 & 67 & --- & 334 & 78 & 18 & 0.0 & 4.8 & 10 & 0.6 \\
\hline SD-7/1952.6/CHn/PP & $\mathrm{i}$ & 595.15 & $05-16-97$ & 8.8 & 980 & 0 & 0.3 & 251 & 67.4 & --- & 353 & 120 & 22 & 0.0 & 6.7 & 11 & 0.9 \\
\hline SD-7/2088.2-2088.5/PP & j & 636.54 & $03-25-97$ & 9.3 & 720 & 0 & 0 & 173 & 54.3 & $-\cdots$ & 157 & 101 & 15 & 0.1 & 4.0 & 25 & 3.9 \\
\hline SD-7/2170.1-2170.5/PP/BT & k & 661.51 & $03-27-97$ & 9.2 & 670 & 0 & 0 & 164 & 55.3 & -- & 100 & 103 & 18 & 0.0 & 3.8 & 18 & 8.4 \\
\hline SD-7/2596.1-2596.3/BF & m & 791.28 & $08-23-96$ & 9.1 & 670 & 0.6 & 0.2 & 158 & 64.3 & -- & 79 & 65 & 83 & 0.1 & 3.2 & 12 & 6.4 \\
\hline SD-7/2596.5-2596.9/BF & $\mathrm{n}$ & 791.47 & $08-21-96$ & 9.0 & 660 & 0 & 0 & 148 & 53.2 & --- & 95 & 71 & 46 & 0.0 & 2.2 & 12 & 7.7 \\
\hline SD-7/2598.3-2598.5/BF & $\mathrm{p}$ & 791.95 & $08-15-96$ & 8.8 & 630 & 0.9 & 0.0 & 152 & 94.8 & --- & 231 & 59 & 22 & 0.1 & 4.0 & 18 & -1.2 \\
\hline $\mathrm{SD}-9 / 94.2-94.4 / \mathrm{PTn} / \mathrm{BT}$ & 1 & 28.7 & $07-08-96$ & 6.2 & 1,050 & 125 & 24 & 43 & 74 & -- & 37 & 0 & 170 & 0.9 & 11 & 260 & -4.3 \\
\hline $\mathrm{SD}-9 / 114.1-114.3 / \mathrm{PTn} / \mathrm{YM}$ & 2 & 34.81 & $03-13-97$ & 6.7 & 950 & 95 & 18 & 55 & 62.2 & -- & 31 & 0 & 138 & 0.3 & 10.9 & 181 & 1.6 \\
\hline SD-9/135.1-135.3/PTn/YM & 3 & 41.21 & $03-11-97$ & 7.5 & 890 & 91 & 15 & 66 & 60.0 & -- & 37 & 0 & 144 & 0.3 & 12.0 & 162 & 2.5 \\
\hline $\mathrm{SD}-9 / 154.0-154.2 / \mathrm{PTn} / \mathrm{BT}$ & 4 & 46.97 & $07-26-96$ & 6.9 & 650 & 72 & 13 & 36 & 55 & -- & 90 & 0 & 93 & 0.9 & 4.7 & 106 & -1.1 \\
\hline SD-9/176.2-176.4/PTn/PV & 5 & 53.74 & $03-07-97$ & 7.5 & 720 & 43 & 9.5 & 95 & 58.5 & --- & 122 & 0 & 64 & 0.1 & 1.9 & 124 & 4.7 \\
\hline $\mathrm{SD}-9 / 251.8-252.0 / \mathrm{PT} / \mathrm{BT}$ & 6 & 76.78 & $02-10-97$ & 7.2 & 510 & 44 & 8.0 & 53 & 55.0 & -- & 92 & 0 & 60 & 0.0 & 14.2 & 73 & 8.2 \\
\hline
\end{tabular}


Table 4. Chemical composition of pore-water samples from boreholes USW SD-7, SD-9, and SD-12, Yucca Mountain area-Continued

[m, meter; $\mu \mathrm{S} / \mathrm{cm}$, microsiemens per centimeter at 25 degrees Celsius; $\mathrm{mg} / \mathrm{L}$, milligrams per liter; --, data not available; 0 , values below detection limit; -, less than; N/S, not sampled; charge balance, (meq cation - meq anion)/(meq cation + meq anion) × 100; PTn, Paintbrush nonwelded; CHn, Calico Hills nonwelded; PP, Prow Pass Tuff; BT, bedded tuff; TSw, Topopah Spring Tuff; YM, Yucca Mountain Tuff]

\begin{tabular}{|c|c|c|c|c|c|c|c|c|c|c|c|c|c|c|c|c|c|}
\hline $\begin{array}{c}\text { Sample } \\
\text { identitication }\end{array}$ & $\begin{array}{c}\text { Symbol } \\
\text { in Piper } \\
\text { diagram } \\
\text { fig. } 5\end{array}$ & $\begin{array}{c}\text { Average } \\
\text { depth } \\
\text { (m) }\end{array}$ & Date & $\mathbf{p H}$ & $\begin{array}{c}\text { Specific } \\
\text { conduc- } \\
\text { tance } \\
(\mu \mathrm{S} / \mathrm{cm})\end{array}$ & $\begin{array}{c}\text { Calcium } \\
\text { Ca } \\
(\mathrm{mg} / \mathrm{L})\end{array}$ & $\begin{array}{c}\text { Magne- } \\
\text { sium } \\
\mathrm{Mg} \\
(\mathrm{mg} / \mathrm{L})\end{array}$ & $\begin{array}{c}\text { Sodium } \\
\mathrm{Na} \\
\text { (mg/L) }\end{array}$ & $\begin{array}{c}\text { Silica } \\
\mathrm{SiO}_{2} \\
(\mathrm{mg} / \mathrm{L})\end{array}$ & $\begin{array}{c}\text { Potas- } \\
\text { sium } \\
\text { K } \\
(\mathrm{mg} / \mathrm{L})\end{array}$ & $\begin{array}{c}\text { Bicar- } \\
\text { bonate } \\
\mathrm{HCO}_{3} \\
(\mathrm{mg} / \mathrm{L})\end{array}$ & $\begin{array}{c}\text { Car- } \\
\text { bonate } \\
\mathrm{CO}_{3} \\
\text { (mg/L) }\end{array}$ & $\begin{array}{c}\text { Chlo- } \\
\text { rine } \\
\text { Cl } \\
\text { (mg/L) }\end{array}$ & $\begin{array}{c}\text { Bromine } \\
\text { Br } \\
(\mathrm{mg} / \mathrm{L})\end{array}$ & $\begin{array}{c}\text { Nitrate } \\
\mathrm{NO}_{3} \\
(\mathbf{m g} / \mathrm{L})\end{array}$ & $\begin{array}{c}\text { Sulfate } \\
\mathrm{SO}_{4} \\
(\mathrm{mg} / \mathrm{L})\end{array}$ & $\begin{array}{l}\text { Charge } \\
\text { balance }\end{array}$ \\
\hline SD-9/1452.6-1452.8/TSw & 7 & 442.78 & $08-30-95$ & 7.5 & 520 & 6.9 & 0 & 112 & 62.5 & -- & 256 & 0 & 15.7 & 0.1 & 10.6 & 62.5 & 1.4 \\
\hline SD-9/1535.2-1535.4/CHn & 8 & 467.96 & 09-07-95 & 7.4 & 530 & 0.8 & 0.1 & 112 & 54.9 & 7 & 226 & 0 & 15.6 & 0.9 & 10.6 & 15.5 & 4.7 \\
\hline SD-9/1619.9-1661.4/CHn & 9 & 500.09 & $01-26-96$ & 8.9 & 610 & 0.4 & 0 & 136.6 & 55.6 & 4 & 232 & 12 & 50.2 & 0.1 & 9 & 18.3 & -0.7 \\
\hline SD-9/1661.1-1661.3/CHn & $\mathrm{X}$ & 506.33 & $01-22-96$ & 9.1 & 670 & 0.7 & 0.0 & 164 & 48.8 & --- & 317 & 0 & 42.0 & 0.1 & 8.2 & 18.9 & 1.9 \\
\hline SD-9/1741.0-1741.2/CHn & $\mathrm{Y}$ & 530.69 & $07-18-96$ & 9.1 & 520 & 0.2 & 0 & 125 & 52 & --- & 185 & 41 & 19 & 1.1 & 4.4 & 10 & 2.2 \\
\hline SD-9/1741.7-1741.9/CHn & - & 530.90 & $07-12-96$ & 8.8 & 310 & 0.2 & 0 & 74 & 53 & --- & 113 & 12 & 15 & 0.2 & 3.8 & 8.7 & 5.0 \\
\hline $\mathrm{SD}-9 / 1800.8 / \mathrm{CHn} / \mathrm{BT}$ & $\mathbf{Z}$ & 548.88 & $01-30-96$ & 9.3 & 790 & 0.8 & 0 & 180.7 & 59.6 & 6 & 137 & 106 & 32.3 & 0.4 & 4.6 & 20.9 & 5.6 \\
\hline $\mathrm{SD}-12 / 265.8-266.1 / \mathrm{PTn} / \mathrm{BT}$ & A & 81.08 & $10-27-95$ & 7.2 & 470 & 48.9 & 7.6 & 28.6 & 62.7 & 3 & 107 & 0 & 49.6 & 0.9 & 15.9 & 47.5 & -0.1 \\
\hline $\mathrm{SD}-12 / 278.6-278.8 / \mathrm{PTn} / \mathrm{pc}$ & B & 84.95 & $06-20-97$ & 7.3 & 580 & 74 & 12.7 & 27 & 71.2 & -- & 159 & 0 & 46 & 0.2 & 16.0 & 75 & 1.7 \\
\hline SD-12/296.1-296.6/TSnw & $\mathrm{C}$ & 90.34 & $09-10-96$ & 7.1 & 490 & 75 & 8.0 & 21 & 72.2 & $\ldots$ & 163 & 0 & 60 & 0.2 & 18.0 & 21 & 2.2 \\
\hline SD-12/1460.7-1461.0/CHn & $\mathrm{D}$ & 445.28 & $04-17-96$ & 8.5 & 490 & 31 & 0 & 89 & 65.5 & -- & 98 & 30 & 55.9 & 0.1 & 7.3 & 30.1 & 4.7 \\
\hline SD-12/1495.5-1495.8/CHn & $\mathrm{E}$ & 455.89 & $06-24-97$ & 8.5 & 550 & 16 & 0.2 & 108 & 87.3 & -- & 49 & 57 & 57 & 0.3 & 8.1 & 55 & -0.7 \\
\hline SD-12/1517.0-1517.4/CHn & $\mathrm{F}$ & 462.44 & $02-21-96$ & 8.3 & 660 & 14 & 0 & 150 & 56.9 & --- & 323 & 0 & 35.6 & 0.1 & 6.4 & 22.5 & 2.5 \\
\hline SD-12/1558.9-1559.5/CHn & G & 475.24 & $08-01-97$ & 8.5 & 640 & 4.4 & 0.1 & 155 & 76.1 & -- & 210 & 41 & 33 & 0.2 & 0.4 & 54 & 0.7 \\
\hline SD-12/1582.5-1582.7/CHn & $\mathbf{H}$ & 482.38 & $08-05-97$ & 8.5 & 420 & 1.2 & 0.1 & 97 & 71.0 & -- & 113 & 29 & 17 & 0.1 & 4.5 & 27 & 4.4 \\
\hline SD-12/1600.6-1603.0/CHn & I & 488.23 & $05-08-96$ & 9.1 & 540 & 2.6 & 0 & 129 & 65.7 & -- & 79 & 90 & 27.8 & 0.1 & 2.6 & 12.6 & 3.2 \\
\hline $\mathrm{SD}-12 / 1636.9 / \mathrm{CHn} / \mathrm{BT}$ & $\mathrm{J}$ & 498.93 & $03-22-96$ & 9.0 & 535 & 3.8 & 0 & 129 & 64.6 & $\cdots$ & 67 & 102 & 15.2 & 0 & 1.7 & 11.2 & 5.6 \\
\hline $\mathrm{SD}-12 / 1901.5 / \mathrm{CHn} / \mathrm{PP}$ & $\mathbf{K}$ & 579.55 & $09-16-96$ & 9.1 & 470 & 1.3 & 0 & 122 & 90.7 & $\cdots$ & 151 & 59 & 22 & 0.1 & 5.7 & 9.3 & 0.2 \\
\hline SD-12/1938.8/CHn/PP & $\mathrm{L}$ & 590.93 & $02-28-96$ & 8.8 & 625 & 1.4 & 0 & 165 & 55.0 & $\cdots$ & 171 & 66 & 34.0 & 0.1 & 1.0 & 26.1 & 5.2 \\
\hline SD-12/1942.4/CHn/PP & $\mathbf{M}$ & 592.04 & $03-11-96$ & 9.1 & 520 & 1.3 & 0 & 140 & 60.1 & $-\cdots$ & 134 & 66 & 24.0 & 0.1 & 3.5 & 24.4 & 4.3 \\
\hline
\end{tabular}




\section{Explanation}

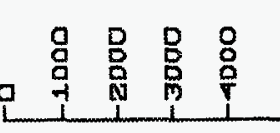

Totol Dieenlyad Sol ida Porte Par Million)

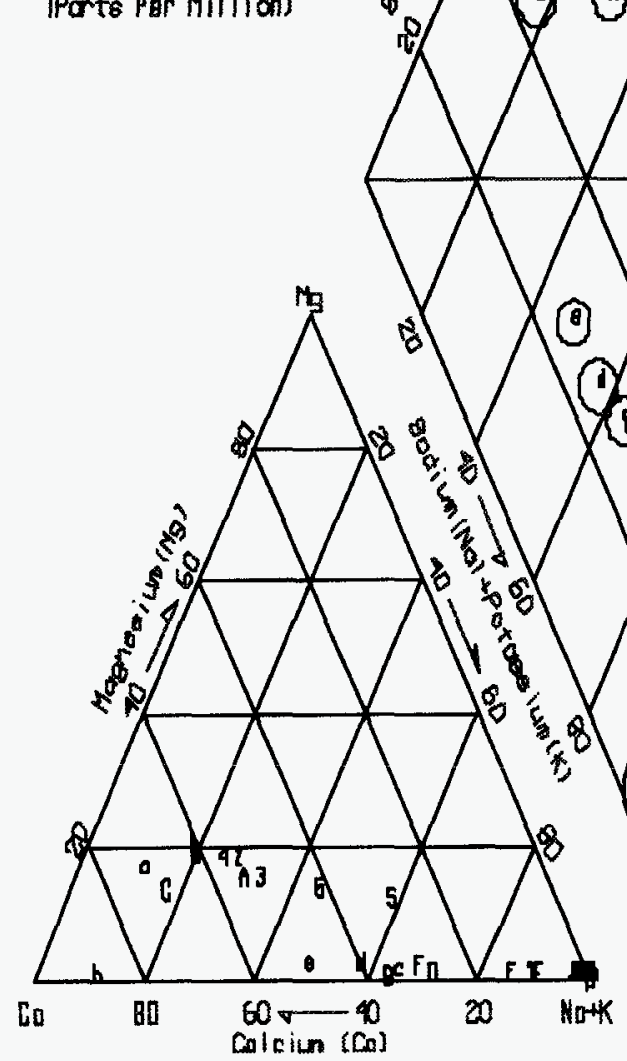

C A T ION S

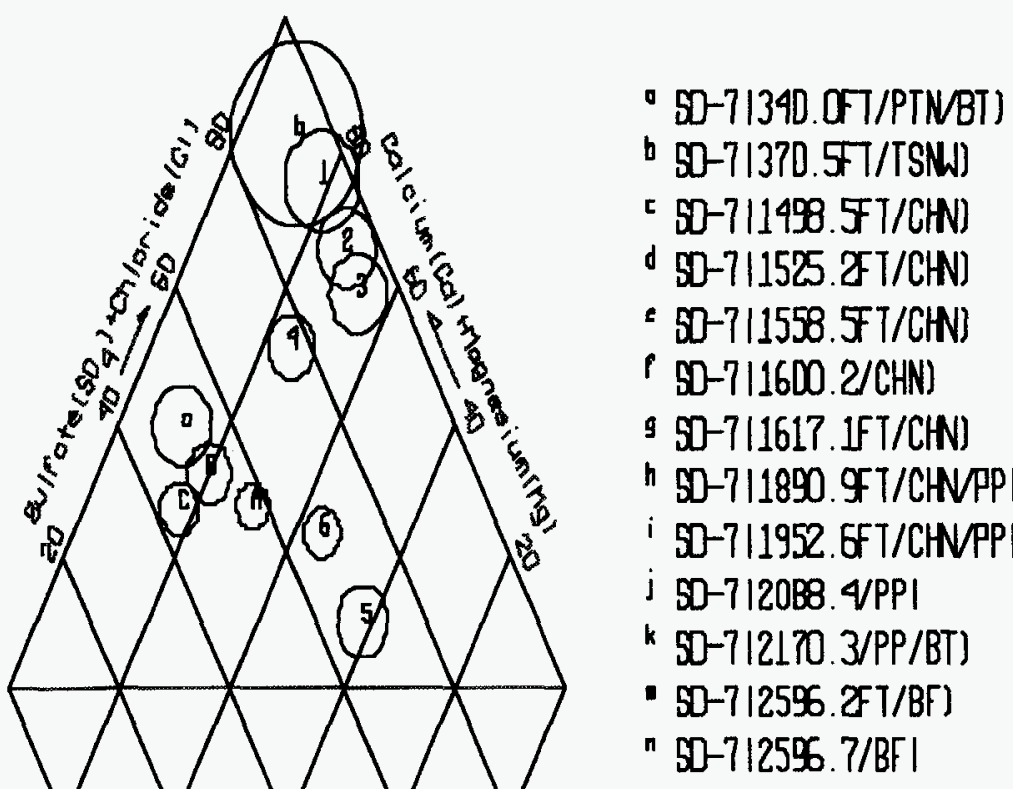

n $50-7125507 / B F 1$
P SD-7(2598. 4FT/EF)

I $\mathrm{gD}-9194.3 F T / P T N B T I$

2 SD-9(114.2/PTNYM)

${ }^{3}$ SD-9(135.2/PINYM)

${ }^{4} \mathrm{gD}-9(154$. ITI/PTN/BT)

5 SD-9(176.3/PINPC)

(5 gD-9(251 9/PINAT)

$I^{7}$ SD-9(1452.7FT/TSH)

S SD-9(1535.3FT/CHN)

9 SD-9(1640.7FT/CHN)

${ }^{x}$ SD-9(1661.2FT/CHN)

${ }^{y}$ SD-9(1741.LFT/CHN)

2 SD-9(1800 .8FT/CHN/BT)
" SD-12 (265. OFT/PTN/BT)

B SD-12(27B.7T/PTN/PC)

c SD-12 (295. 4T/TSNN)

D SD-12(1460.9T/CHN)

E SD-12(1495.7FT/CHN)

F SD-12(1517.2FT/CHN)

SO-12 (1559.2/CHN)

H SD-12 (15B2.6/CHN)

I SD-12(1601.8FT/CHN)

J SD-12 (1636. 9T/CHNVT)

${ }^{k}$ SD-12(1901.5FT/CHN/PP)

L SD-12 (193B.8FT/CHN/PP)

" SD-12 (1942.4T/CHN/PP)

$\%$ meq $/$ l = percentage of

milliequivalents per liter

Figure 5. USW SD-7, SD-9, and SD-12 pore-water compositions. 
In all tables in this paper, core samples are identified as "UZX-YYYY-ZZZZ/UPN/" or "UZX-YYYY-ZZZZ /T/UPN/D". The first three letters "UZX" are used to identify the unsaturatedzone borehole number; for example UZ-14. The next group of letters YYYY-ZZZZ indicates the depth interval (in feet unless noted otherwise) where core was obtained; UPN stands for uniaxial compression first stage (UP1) or second pressure stage (UP2), and so on; " $D$ " stands for distillation; and " $T$ " stands for imbibed test water (not the original pore water). For example, a sample identification of "......../UP1/D" indicates a vacuum-distilled water sample from core that has undergone first-stage uniaxial compression. If there is no "UPN" in front of "D" (such as "UZ14YYYY-ZZZZ/D"), the water was extracted by vacuum distillation without compression.

Chemical compositions of water samples show clear variations. Locations of UZ-14, SD-9, and the NRG boreholes are in the northern part of Yucca Mountain, while boreholes SD-12 and SD-7 are in the southern part of the mountain. The pore waters extracted from bedded tuff are calcium-chloride- or calcium-sulfate-type waters which plot near the top part of the diamond in a Piper-type diagram (see figure 3, for example). Three samples from Topopah Spring Tuff, one from each borehole, are shown in figure 5. Two of them, "b" (SD-7) and "C" (SD-12), are from the upper nonwelded unit and one, "7". (SD-9), is from the lower welded unit of the Topopah Spring Tuff. Chemical composition of upper and lower units also are different, with the upper unit closer to calciumchloride and calcium-sulfate type, and the lower unit closer to sodium-bicarbonate type. Chloride concentration in the lower unit is less concentrated than the upper unit, indicating dilution by the fracture water. The pore waters extracted from the Calico Hills nonwelded hydrogeologic unit are sodium-carbonateor sodium-bicarbonate-type waters which plot near the lower part of the diamond. Samples represented by "c", "d", "e", "f", "g", "D" and "E" in figure 5 are pore waters from borehole SD-7 and SD-12 in the Calico Hills nonwelded unit. These waters tend toward calcium-chloride- and calcium-sulfate-type waters instead of the sodium-bicarbonate- or sodiumcarbonate-type waters typical of the Calico Hills nonwelded unit in the other boreholes. This could be due to less $\mathrm{Ca} / \mathrm{Na}$ cation exchange, with less sodium ion released into water in the SD-7 and SD- 12 boreholes. Therefore, in spite of large distances separating these boreholes, chemical compositions are, with few exceptions which will be described below, in general similar and vary according to the lithologic units in which they were obtained.

The dissolved solids concentrations of the porewater samples from the Yucca Mountain Tuff at $13.78 \mathrm{~m}$ and from the Prow Pass Tuff at $614.11 \mathrm{~m}$ (table 2), at $615.85 \mathrm{~m}$, and at $617.28 \mathrm{~m}$ in UZ-14 (shown by points $1, \mathrm{f}, \mathrm{g}$ and $\mathrm{h}$ in figure 3 ) have very high concentrations of dissolved solids (about $1,200 \mathrm{mg} / \mathrm{L}$ for the $13.78-\mathrm{m}$ sample and $2,000 \mathrm{mg} / \mathrm{L}$ for samples in the Prow Pass Tuff). Also, pore-water sample $\mathrm{G}$ in figure 4 from the NRG-6 borehole at $78.03 \mathrm{~m}$ has a total dissolved solids concentration of $3,500 \mathrm{mg} / \mathrm{L}$. Evaporative concentration and/or extended water-rock interactions can result in such a large concentration. If the waters were concentrated by evaporation, one possible mechanism could involve evaporation by vapor transport in the unsaturated zone.

The sample from UZ-14 at a depth of $13.78 \mathrm{~m}$ (UZ14/45.0-45.4/PTn/YM in table 2) has $\mathrm{Na}$ and $\mathrm{Cl}$ concentrations of $249.3 \mathrm{mg} / \mathrm{L}$ and $245 \mathrm{mg} / \mathrm{L}$, respectively. Samples at slightly greater depth, such as UZ14/85.2-85.6/up 1UZ14/91.0-91.3/up1, and UZ14/95.5-95.9/up1 (table 3, Yang and others, 1996), have an average $\mathrm{Na}$ concentration of $39 \mathrm{mg} / \mathrm{L}$ and $\mathrm{Cl}$ concentration of $60 \mathrm{mg} / \mathrm{L}$. Sample UZ14/45.045.4/PTn/YM is more concentrated in $\mathrm{Na}$ by a factor of 6 and in $\mathrm{Cl}$ by a factor of 4 than the latter. If evaporative concentration is the cause, both $\mathrm{Na}$ and $\mathrm{Cl}$ should be elevated by the same factor, a relation that is not observed. Further, the stable-isotope composition of this pore water ( $\delta \mathrm{D}$ and $\delta^{18} \mathrm{O}$ values) plots close to the Yucca Mountain Precipitation Line (YMPL) in the $\delta \mathrm{D}$ versus $\delta^{18} \mathrm{O}$ diagram (see fig. 19) indicating little moisture loss by evaporation. (A detailed explanation is given later in the section on the interpretation of stable-isotope data.) These two lines of evidence indicate that high total dissolved solids in this sample are the result of water-rock interactions with only a minor contribution from evaporative concentration. Similarly, for samples from the Prow Pass Tuff from UZ-14 and the NRG-6 sample from a 78.0-m depth, the concentration factors for $\mathrm{Na}$ and $\mathrm{Cl}$ are different, again indicating extensive water-rock interaction. The $\delta \mathrm{D}$ and $\delta^{18} \mathrm{O}$ values of these pore waters, when plotted on the $\delta \mathrm{D}$ versus $\delta^{18} \mathrm{O}$ diagram, also show deviation from the YMPL, indicating loss of water by evaporation. Evaporative concentration does occur to some extent (less than 10 percent) but cannot account for the 
observed doubled concentration factor. (See detailed explanation later in the interpretations of stableisotope data.) Water-rock interactions thus appear to be the major cause of high dissolved-solid concentrations, and there is no evidence from these samples for major unsaturated-zone vapor transport.

The concentrations of major ions in the pore water are three to ten times larger than those in the perched or saturated-zone waters. These pore waters are from bedded/nonwelded tuffs of the Paintbrush nonwelded (PTn) and Calico Hills nonwelded (CHn) hydrogeologic units only, not from the large block of the welded Topopah Spring Tuff(TSw) hydrogeologic unit at Yucca Mountain. If the more dilute infiltrating waters provide recharge to the perched or saturatedzone waters, they must flow rapidly through fractures or permeable zones in the intervening PTn or $\mathrm{CHn}$ units to avoid mixing with the chemically concentrated pore waters in those units. The water flux through such a flow regime is small based on the narrow shape of the tritium peaks. This conceptual model is also supported by the detection of post-bomb ${ }^{3} \mathrm{H}$ in the deep unsaturated zone of the mountain, indicating a fast flow path (Yang and others, 1996).

\section{Tritium Profiles in Boreholes}

Pre-1952 tritium concentrations in precipitation were around 10 TU (Davis and Bentley, 1982). In 1954, atmospheric nuclear tests artificially increased the average annual tritium concentration in precipitation near Yucca Mountain to about 200 TU, with a monthly maximum of about 700 TU. By 1963, further nuclear tests in the atmosphere increased the average annual tritium concentration in precipitation to about 2,000 TU, with a monthly maximum of about 4,000 TU (International Atomic Energy Agency reports, 1969-86). After three half-lives of tritium decay from 1954 to 1990 , the average annual tritium concentration in precipitation for 1954 input still remained at $25 \mathrm{TU}$, with a monthly maximum of about 87 TU. For 1963 input, average annual tritium concentration in precipitation after two half-lives from 1963 to 1987 still remained at $500 \mathrm{TU}$ with a monthly maximum of $1,000 \mathrm{TU}$. Therefore, water samples extracted from rock core with tritium concentrations greater than 25 TU in 1990 would indicate mixtures derived from pre- and post-1954 precipitation. Tritium concentrations greater than 500 TU in 1987 would indicate mixtures derived from pre- and post-1963 precipitation. Assuming piston flow is dominant in the unsaturated zone of Yucca Mountain, an assumption described by Yang and others (1996), the high tritium concentrations of pore-water samples such as these can be attributed mainly to post-1954 or post-1963 precipitation.

The purpose of the following interpretations of tritium concentrations is not to determine the age of pore water in the unsaturated zone but to isolate any pore-water samples that could have been derived mainly from post-1954 precipitation. Tritium profiles for pore waters in borehole UZ-14 are shown in figure 6 . Tritium concentrations between 20 to $40 \mathrm{TU}$ are observed in the intervals between 30 to $45 \mathrm{~m}$ in the Pah Canyon Tuff and at $385 \mathrm{~m}$ depth in the Topopah Spring Tuff. Only small intervals of the NRG-6 and NRG-7a cores were analyzed for pore-water tritium and ${ }^{14} \mathrm{C}$. The data are shown in figures $7 \mathrm{~A}$ and $7 \mathrm{~B}$. High tritium concentrations of about 30 to $150 \mathrm{TU}$ in a broad peak are observed in NRG-6 boreholes (fig. 7A) from $53.3 \mathrm{~m}$ to $74.7 \mathrm{~m}$ in the Pah Canyon Tuff and near the top of the Topopah Spring Tuff. One large tritium concentration of about $47 \mathrm{TU}$ is observed at a depth of $108.7 \mathrm{~m}$ in NRG-7a. Tritium data for pore waters extracted from SD-7, SD-9, and SD-12 borehole cores are shown in figures $8,9 \mathrm{~A}$, and $9 \mathrm{~B}$. The tritium concentrations are less than $10 \mathrm{TU}$ in SD-7 samples and as large as $14.3 \mathrm{TU}$ in the $\mathrm{CHn}$ unit of SD-12; SD-9 core samples have tritium concentrations as high as $21 \mathrm{TU}$ at $50-\mathrm{m}$ depth. As mentioned previously, only limited samples were analyzed from each borehole. Therefore, some fast flow paths may not have been detected.

\section{Residence Times of ${ }^{14} \mathrm{CO}_{2}$ and Pore Water}

Gaseous-phase ${ }^{14} \mathrm{C}$ and $\delta{ }^{13} \mathrm{C}$ data from SD-7, SD-9 and SD-12 are shown in table 5. The $\delta^{13} \mathrm{C}$ samples collected by the WG balloon method varied from -10 to -25 per mil in $\delta^{13} \mathrm{C}$ values for the replicate samples. Therefore, these data are not listed here. Problems likely were in the balloon nozzle. Values of $\delta^{13} \mathrm{C}$ values obtained by Desert Research Institute (DRI) are less variable, although they tend to be systematically more negative by a few units per mil when collected with the molecular sieve. The other possible explanation of $\delta^{13} \mathrm{C}$ values less than -20 per mil could be due to Exploratory Study Facility (ESF) ventilation, which causes degassing of pore-waterdissolved $\mathrm{CO}_{2}$ that was collected during gas sampling 


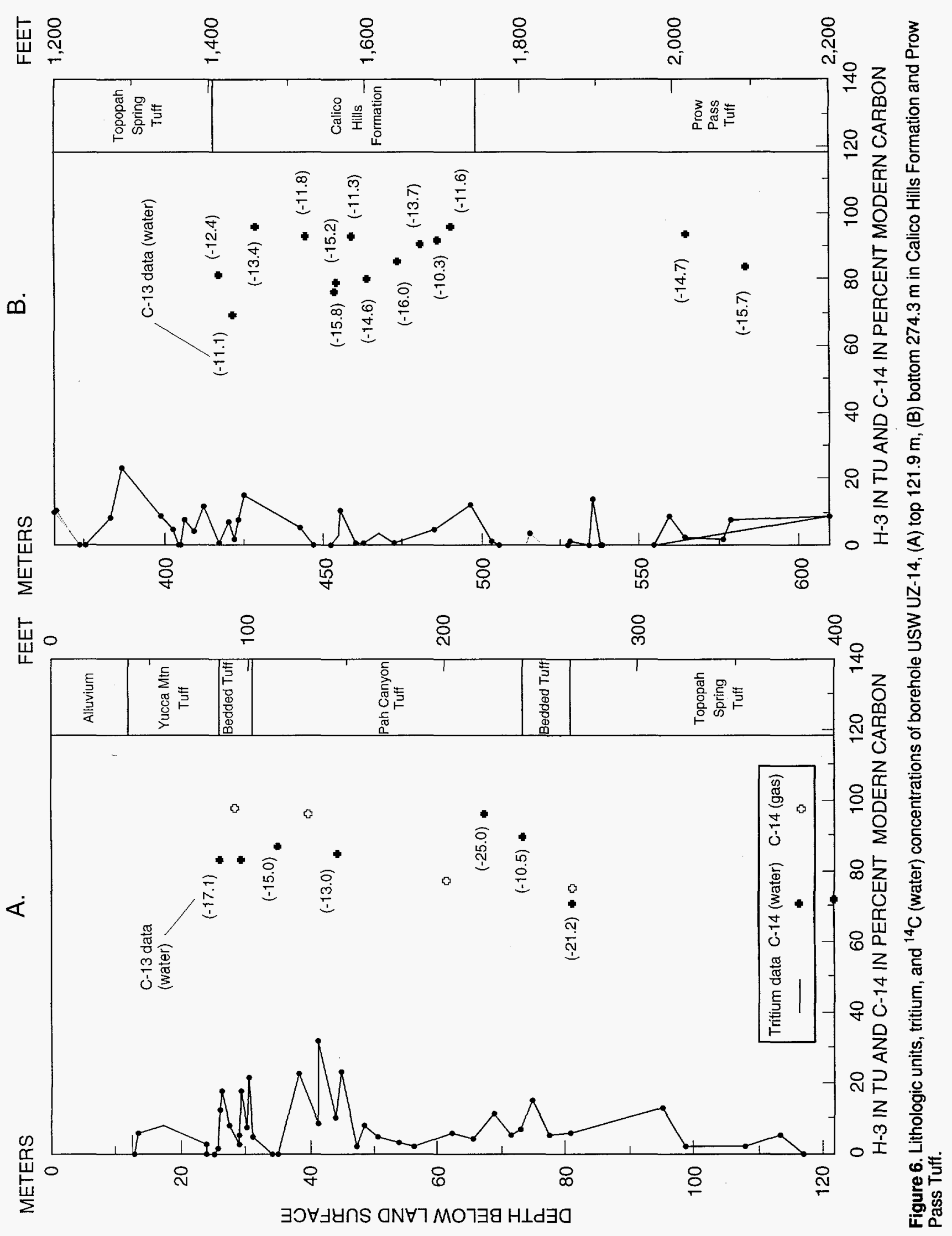




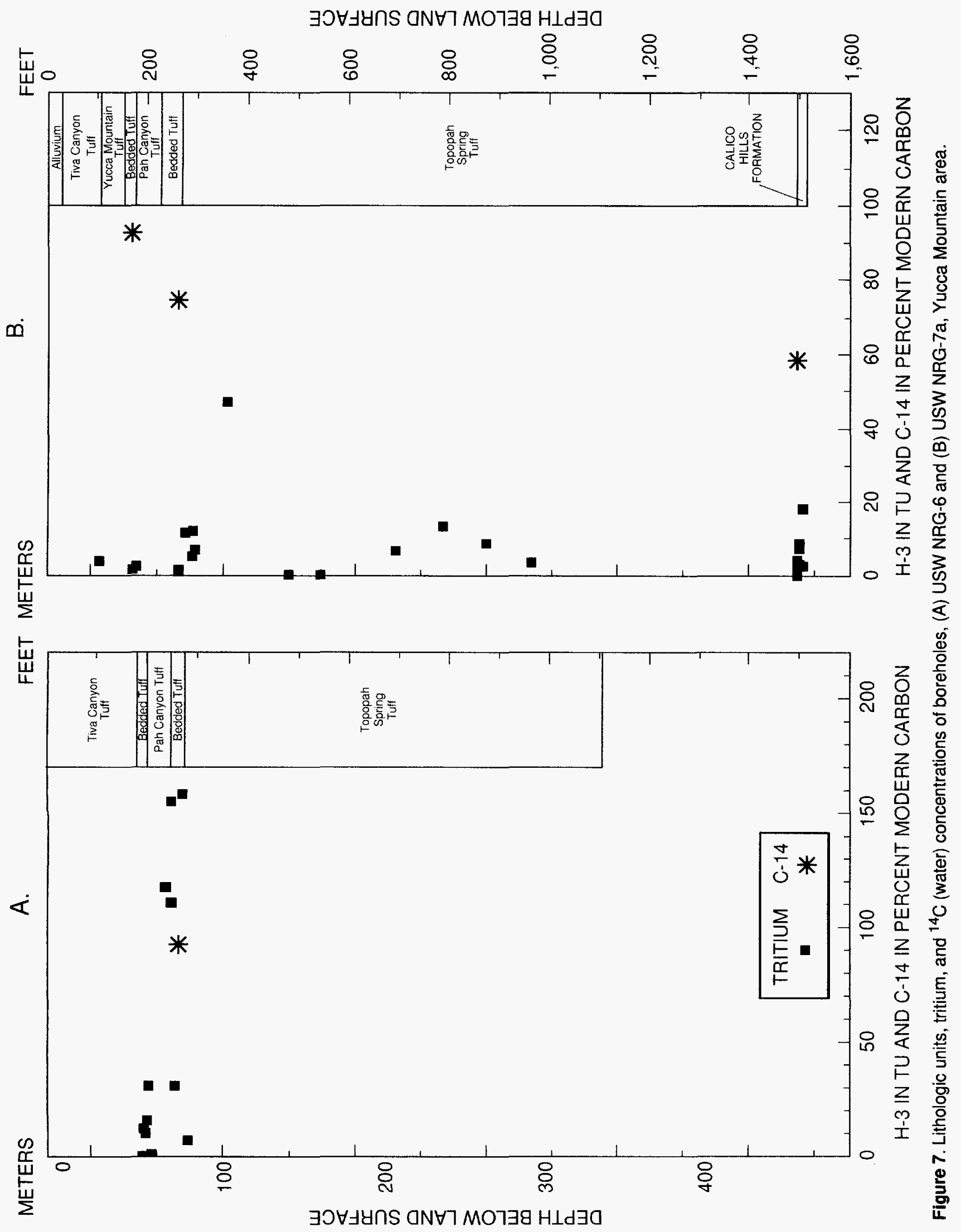




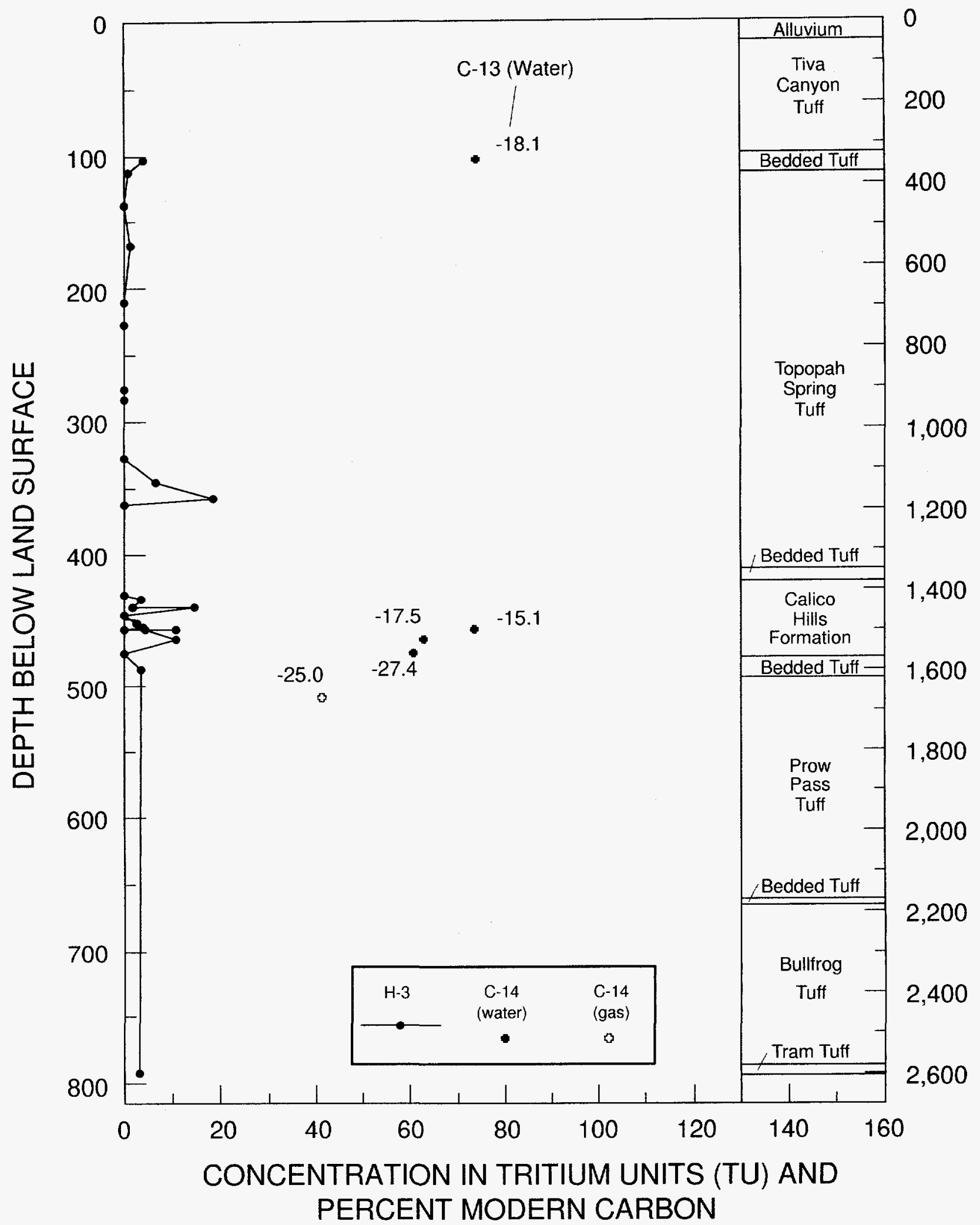

Figure 8. Lithologic units, tritium, and ${ }^{14} \mathrm{C}$ (gas and water) concentrations of borehole USW SD-7, Yucca Mountain area. 


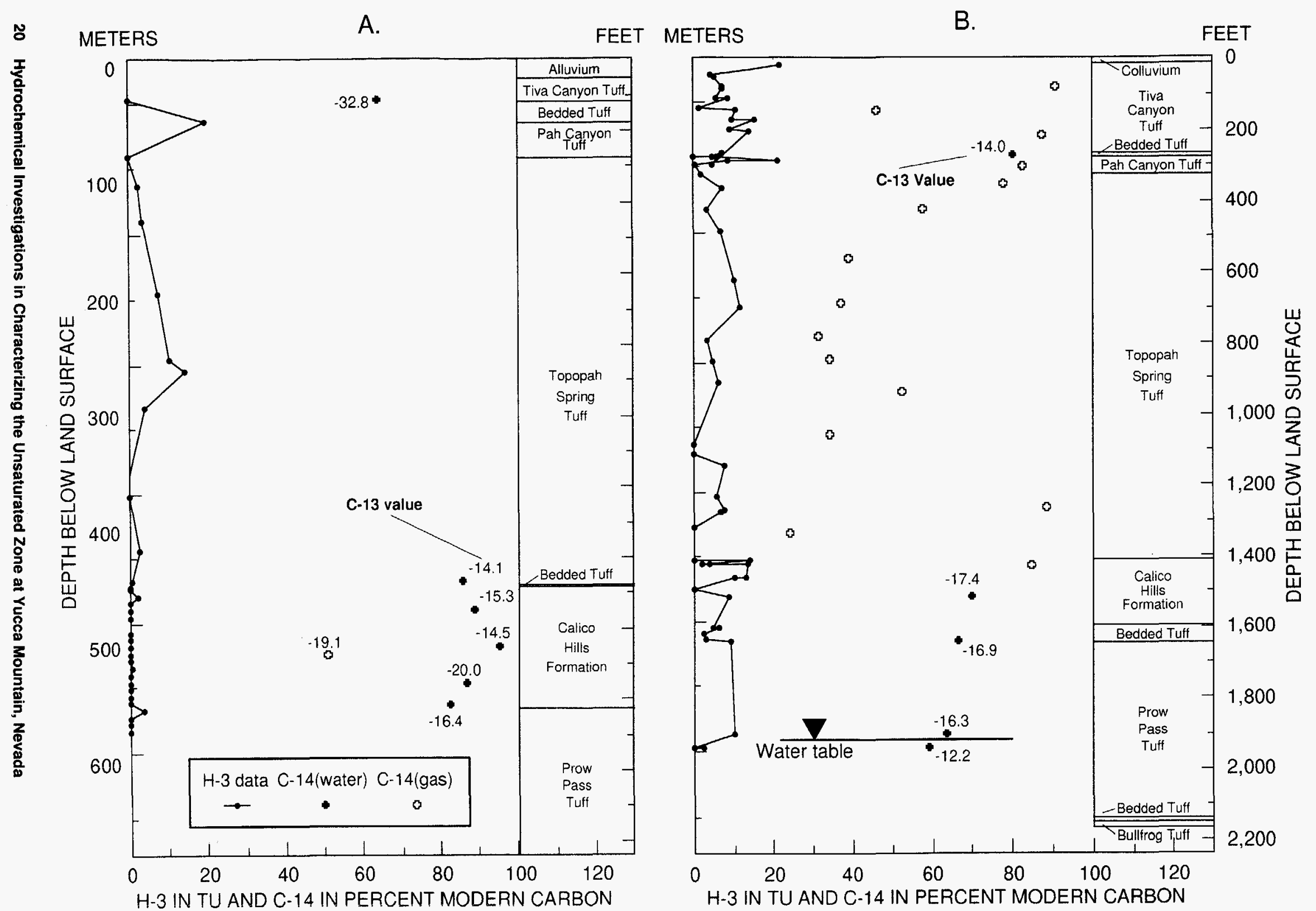

Figure 9. Lithologic units, tritium, and ${ }^{14} \mathrm{C}$ (gas and water) concentrations of boreholes (A) USW SD-9 and (B) SD-12, Yucca Mountain area. 
Table 5. Gaseous-phase carbon- $14\left({ }^{14} \mathrm{C}\right), \delta^{13} \mathrm{C}$ (gas), and fracture-frequency data from boreholes USW SD-7, SD-9, and SD-12, Yucca Mountain area

[m, meter; pmc, percent modern carbon; ---, data not analyzed]

\begin{tabular}{|c|c|c|c|c|c|c|}
\hline Borehole & $\begin{array}{l}\text { Depth } \\
\text { (m) }\end{array}$ & $\begin{array}{l}\text { Station with } \\
\text { instrument }\end{array}$ & Lithologic unit $^{1}$ & $\begin{array}{c}{ }^{14} \mathrm{C} \\
(\mathrm{pmc}) \\
\mathrm{DRl^{2 }}\end{array}$ & $\begin{array}{c}\delta^{13} \mathbf{C} \\
\text { (per mil) } \\
\text { DRI }^{2}\end{array}$ & $\begin{array}{l}\text { Published } \\
\text { 3.05-m } \\
\text { composite }\end{array}$ \\
\hline SD-7 & $508-633$ & -- & Prow Pass Tuff & 41.5 & -25.0 & $\ldots$ \\
\hline SD-9 & $0-450.5$ & --- & Tiva-Topopah Spring Tuff & 96.7 & -16.1 & --- \\
\hline SD-9 & $450.5-537.7$ & --- & Calico Hills nonwelded unit & 51.0 & -19.1 & --- \\
\hline SD-12 & 24.7 & $\mathrm{P}$ & Tiva Canyon Tuff & 91.0 & -22.6 & 8 \\
\hline SD-12 & 43.9 & 0 & Tiva Canyon Tuff & 49.2 & -20.2 & 4 \\
\hline SD-12 & 65.2 & $\mathrm{~N}$ & Tiva Canyon Tuff & 87.4 & -22.0 & 15 \\
\hline SD-12 & 91.7 & $\mathrm{~L}$ & Topopah Spring Tuff & 82.7 & -16.4 & --- \\
\hline SD- 12 & 107.0 & $\mathrm{~K}$ & Topopah Spring Tuff & 77.8 & -21.6 & 5 \\
\hline SD- 12 & 128.9 & $\mathbf{J}$ & Topopah Spring Tuff & 58.0 & -22.1 & 6 \\
\hline SD-12 & 171.0 & I & Topopah Spring Tuff & 39.0 & -23.6 & 5 \\
\hline SD-12 & 208.2 & $\mathrm{H}$ & Topopah Spring Tuff & 36.9 & -24.7 & 18 \\
\hline SD-12 & 236.8 & G & Topopah Spring Tuff & 31.6 & -25.6 & 62 \\
\hline SD-12 & 256.6 & $\mathrm{~F}$ & Topopah Spring Tuff & 34.1 & -24.4 & 6 \\
\hline SD-12 & 285.0 & $\mathrm{E}$ & Topopah Spring Tuff & 52.4 & -21.9 & 13 \\
\hline SD-12 & 322.5 & $\mathrm{D}$ & Topopah Spring Tuff & 34.3 & -23.8 & 35 \\
\hline SD-12 & 385.6 & $\mathrm{C}$ & Topopah Spring Tuff & 88.7 & -22.9 & 48 \\
\hline SD-12 & 407.2 & B & Topopah Spring Tuff & 24.4 & -24.6 & 7 \\
\hline SD-12 & 435.9 & A & Calico Hills nonwelded unit & 84.5 & -17.3 & --- \\
\hline
\end{tabular}

(a kinetic fractionation effect). Carbon dioxide concentrations are not reported here due to variable data, with values ranging from 1,000 to $7,000 \mathrm{ppm}$.

The excess $\mathrm{CO}_{2}$ could come from dissolved $\mathrm{CO}_{2}$ in pore water of the Topopah Spring Tuff. The ${ }^{14} \mathrm{C}$ activities of $\mathrm{CO}_{2}$ gas from boreholes SD-9 and SD-12 are plotted in figures $9 \mathrm{~A}$ and $\mathrm{B}$, and $\delta^{13} \mathrm{C}$ values from SD-12 are plotted in figure 10 . One ${ }^{14} \mathrm{C}$ activity of gaseous-phase $\mathrm{CO}_{2}$ was derived from the interval between the top of the Calico Hills nonwelded unit and the water table of SD-9. The ${ }^{14} \mathrm{C}$ activity of this sample is $51 \mathrm{pmc}$, significantly smaller than the ${ }^{14} \mathrm{C}$ activities of pore waters (between 82.3 and 95.3 $\mathrm{pmc}$ ) in the same zone. If the $\mathrm{CO}_{2}$ in the gaseous phase were in isotopic equilibrium with $\mathrm{CO}_{2}$ in the aqueous phase of the pore water, the ${ }^{14} \mathrm{C}$ activities in the two phases should be comparable. The fact that they are different possibly could be due to atmospheric ${ }^{14} \mathrm{CO}_{2}$ contamination to the pore water during drilling.

The ${ }^{14} \mathrm{C}$ activities of gaseous-phase $\mathrm{CO}_{2}$ in instrumented borehole SD-12 (table 5) decreased from surface to depth with some irregularly large ${ }^{14} \mathrm{C}$ activities in stations $\mathrm{E}, \mathrm{C}$, and $\mathrm{A}$ and small ${ }^{14} \mathrm{C}$ activity in station $\mathrm{O}$. Anomalously large ${ }^{14} \mathrm{C}$ activities in station $\mathrm{C}$ and $\mathrm{A}$ may indicate contamination of the samples. Samples from station $\mathrm{C}$ may have been affected by leakage through the borehole instrumentation support pipe (J.P. Rousseau, U.S. Geological Survey, oral commun., 1997). Station A lies in a region of strongly low pressure at depth (identified from monitoring sensors) with complete attenuation of the surface (synoptic) barometric-pressure signal. Gas-monitoring evidence indicates that station A likely was contaminated with atmospheric air drawn in due to the low pressure, prior to the instrumentation and sealing of the borehole (J.P. Rousseau, U.S. Geological Survey, oral commun., 1997). Moderately large ${ }^{14} \mathrm{C}$ activity at station $\mathrm{E}$ may be related to the relatively high fracture frequency noted at that station (table 5). The majority of ${ }^{14} \mathrm{C}$ activities in gas-phase $\mathrm{CO}_{2}$ from SD-12, however, are consistent with the data obtained from borehole UZ-1 and further are consistent with gas 


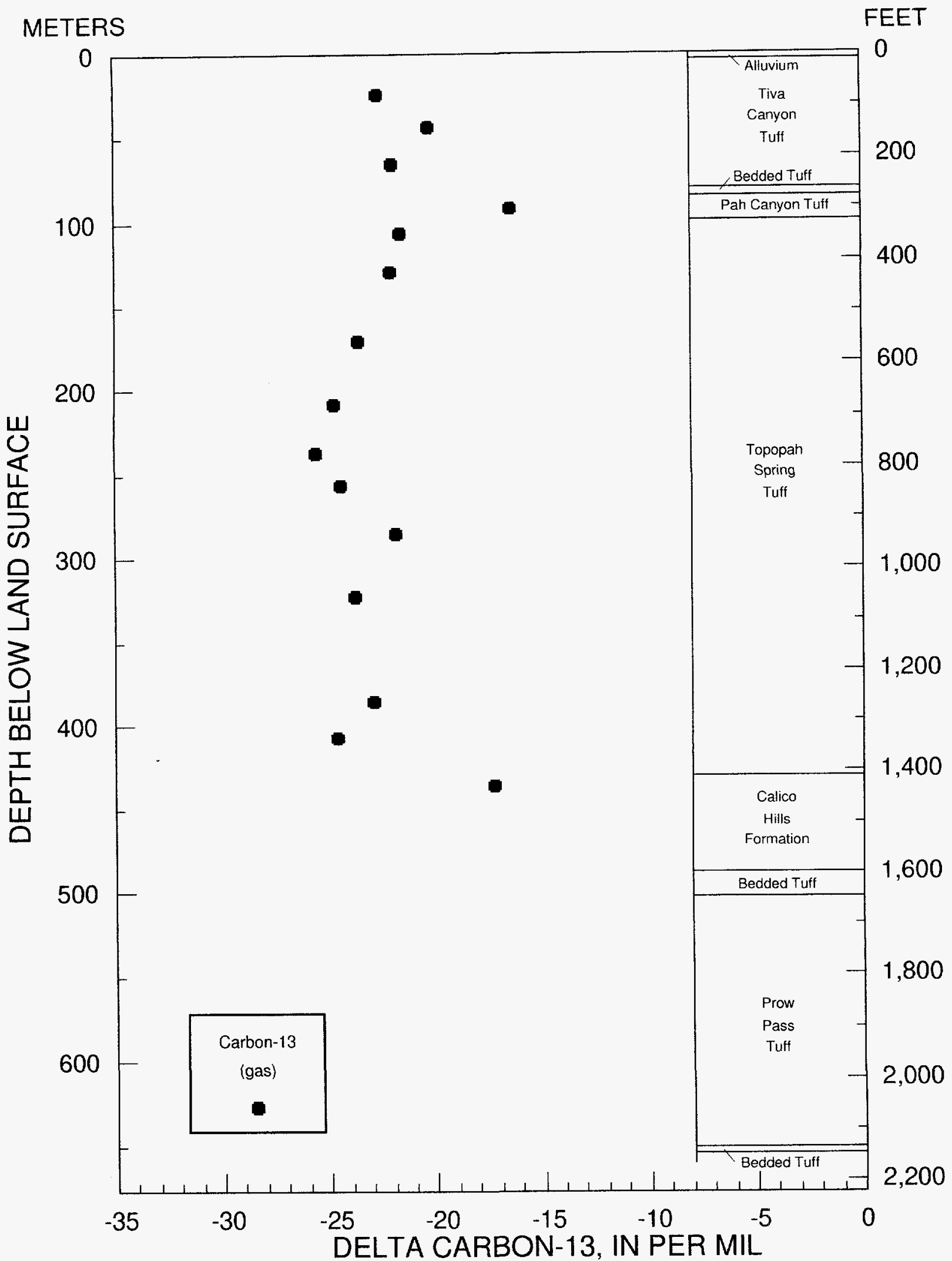

Figure 10. Lithologic units and $\delta^{13} \mathrm{C}$ (gas) values, borehole USW SD-12, Yucca Mountain area. 
transport by a postulated diffusion mechanism as previously concluded (Yang and others, 1996).

Yang and others (1996) stated that the high pore-water ${ }^{14} \mathrm{C}$ pmc values in the $\mathrm{CHn}$ from borehole UZ-14 could be related to (1) ${ }^{14} \mathrm{CO}_{2}$ in the gaseous phase possibly exchanging with the bicarbonate species in the pore water; and/or (2) the large amounts of cement used below a perched-water zone at $383.1 \mathrm{~m}$ to seal off the borehole wall and prevent perchedwater leakage (Portland cement contains $\mathrm{Ca}(\mathrm{OH})_{2}$ which will absorb atmospheric $\mathrm{CO}_{2}$ during mixing with water and release the $\mathrm{CO}_{2}$ during exothermic curing). In more recent pore-water data obtained from the Calico Hills nonwelded unit in SD-9, ${ }^{14} \mathrm{C}$ values ranged from $82.3 \mathrm{pmc}$ to $95.3 \mathrm{pmc}$ between depths of $467.9 \mathrm{~m}$ and $548.6 \mathrm{~m}$. Those data are in the same range of ${ }^{14} \mathrm{C}$ values obtained in the Calico Hills Formation from UZ-14. Both boreholes are located in the northern part of Yucca Mountain; SD-9 was drilled without using cement. Therefore, it is unlikely that the ${ }^{14} \mathrm{C}$ data in the Calico Hills nonwelded unit from UZ-14 were contaminated by cement.

With regard to exchange of young ${ }^{14} \mathrm{C}$ in gaseous-phase $\mathrm{CO}_{2}$ with the bicarbonate species in the pore water in the Calico Hills nonwelded unit of UZ14 and SD-9, the sparse data obtained so far are inconclusive. Gaseous-phase ${ }^{14} \mathrm{C}$ data (table 5) from one analysis from the Calico Hills nonwelded unit in SD-9 show 51.0 pmc (calculated age about 5,800 years), but one analysis from the Calico Hills nonwelded unit in SD-12 shows 84.5 pmc (about 1,340 years). From the ${ }^{14} \mathrm{C}$ data of gaseous-phase $\mathrm{CO}_{2}$ obtained in the UZ-1 and SD-12 boreholes, true gaseous-phase ${ }^{14} \mathrm{C}$ activities likely are about $50 \mathrm{pmc}$ in the Calico Hills nonwelded unit (Yang and others, 1998). A possible explanation for the discrepancy between large ${ }^{14} \mathrm{C}$ activities in the Calico Hills nonwelded unit in UZ-14 and SD-9 is that pore waters were contaminated with atmospheric ${ }^{14} \mathrm{CO}_{2}$ during drilling or mixing of young water with old water. However, the fact that ${ }^{14} \mathrm{C}$ activities in the Calico Hills nonwelded unit in SD-7 and SD-12 are about 40 to $85 \mathrm{pmc}$ would exclude large contamination of cores by the drilling air (with ${ }^{14} \mathrm{C}$ about $120 \mathrm{pmc}$ ) because all these boreholes were drilled with air. The $\delta^{13} \mathrm{C}$ values of bicarbonate carbon in pore water from the Calico Hills nonwelded unit in SD-9, UZ-14, SD-7, and SD-12 also do not show large contamination from atmospheric ${ }^{14} \mathrm{CO}_{2}\left(\delta^{13} \mathrm{C}\right.$ of -7 to -8 per mil in atmospheric $\mathrm{CO}_{2}$ ). For confirmation of positive ${ }^{14} \mathrm{C}$ values in pore water, ${ }^{14} \mathrm{C}$ analyses should be made on cores that are drilled with compressed nitrogen gas (which contains no carbon dioxide as a coolant) instead of atmospheric air. In contrast, perched water encountered in boreholes likely is uncontaminated by the ${ }^{14} \mathrm{CO}_{2}$ in the drilling air because the mass of carbon in these water reservoirs is orders of magnitude larger than in the drilling air.

\section{STABLE-ISOTOPE COMPOSITIONS ( $\delta \mathrm{D}$ AND $\delta^{18}$ O) OF PORE WATER FROM USW UZ-14}

Oxygen, deuterium $\left({ }^{2} \mathrm{H}\right)$, and hydrogen isotopic ratios of water have great value in hydrologic studies because they are intimately dependent upon the nature of physical processes that govern water transport (Craig, 1961b; Gat, 1980). Stable-isotope ratios $\left({ }^{18} \mathrm{O} /{ }^{16} \mathrm{O}\right.$ and $\left.{ }^{2} \mathrm{H} /{ }^{1} \mathrm{H}\right)$ expressed as $\delta^{18} \mathrm{O}$ and $\delta \mathrm{D}$ can be used to indicate the origins of water, climatic and evaporative history of water, and flow paths through the unsaturated zone. They can also be used as indicators of transport properties or water interactions with other minerals. The lack of long-term direct measurements of infiltration requires proxy indicators of water movement through the unsaturated zone to extend the record into the past. The isotopes most commonly used in hydrology are those that are constituents of water molecules $\left({ }^{18} \mathrm{O},{ }^{2} \mathrm{H}\right.$, and $\left.{ }^{3} \mathrm{H}\right)$. Other environmental isotopes $\left({ }^{14} \mathrm{C},{ }^{13} \mathrm{C}\right.$, and $\left.{ }^{36} \mathrm{Cl}\right)$ that occur in dissolved compounds are also valuable for studying ground-water cycles (Fontes, 1980) and have been applied in the previous section.

Hydrogen has two stable isotopes $\left({ }^{2} \mathrm{H}\right.$ and $\left.{ }^{1} \mathrm{H}\right)$, and oxygen has three stable isotopes $\left({ }^{16} \mathrm{O},{ }^{17} \mathrm{O}\right.$, and ${ }^{18} \mathrm{O}$ ). Because hydrogen and oxygen are components of the water molecule, isotopic fractionation of both is usually co-variant. Consequently, the isotope ratios of these two elements generally are discussed together. Natural variations of the abundance of these stable isotopes can be related to the history of the water molecules (Gat and Gonfiantini, 1981). Rather than express these variations as the absolute abundance of the isotopes, Vienna standard mean ocean water (VSMOW) is used as the standard against which the natural variations are measured. 
Thus:

$$
\begin{gathered}
\delta^{18} \mathrm{O}(\text { per mil })=\left[\left(\mathrm{R}_{\mathrm{o}}-\mathrm{R}_{\text {std,o }}\right) / \mathrm{R}_{\text {std }, \mathrm{o}}\right] \times 10^{3} \\
\delta \mathrm{D}(\text { per mil })=\left[\left(\mathrm{R}_{\mathrm{h}}-\mathrm{R}_{\text {std }, \mathrm{h}}\right) / \mathrm{R}_{\text {std }, \mathrm{h}}\right] \times 10^{3}
\end{gathered}
$$

where $\delta^{18} \mathrm{O}$ (per mil) and $\delta \mathrm{D}$ (per mil) are the deviation in ${ }^{18} \mathrm{O}$ and $\mathrm{D}$ of the sample from the reference standard in units of per mil respectively, $\mathrm{R}_{\mathrm{o}}={ }^{18} \mathrm{O} /{ }^{16} \mathrm{O}$ and $\mathrm{R}_{\mathrm{h}}=\mathrm{D} /{ }^{1} \mathrm{H}$ of the sample being studied, and $\mathrm{R}_{\mathrm{std}, \mathrm{o}}=$ ${ }^{18} \mathrm{O} /{ }^{16} \mathrm{O}$ and $\mathrm{R}_{\text {std, } \mathrm{h}}=\mathrm{D} /{ }^{1} \mathrm{H}$ of the VSMOW.

After surface water enters the ground-water system, its original isotopic composition is typically conserved. Some factors, however, can cause the $\delta^{18} \mathrm{O}$ and $\delta \mathrm{D}$ concentrations of unsaturated-zone water and ground water to differ from local meteoric water. These factors include:

1. Recharge from a water body of partially evaporated surface water. Evaporation from surface water bodies is a nonequilibrium process that enriches $\delta^{18} \mathrm{O}$ and $\delta \mathrm{D}$ in the water such that the $\delta \mathrm{D} / \delta{ }^{18} \mathrm{O}$ slope is less than 8 and usually between 3 and 6 .

2. Recharge to an aquifer from an area of different altitude or latitude.

3. Mixing of two or more sources of water.

4. Recharge of either the ground water or UZ water from a different climatic regime in the past.

5. Interaction with aquifer carbonate or silicate rocks at high temperature. Exchange of oxygen in water and rock at high temperatures increases the $\delta^{18} \mathrm{O}$ of the water (the $\delta^{18} \mathrm{O}$ shift).

6. Fractionation between the ground or pore water and mineral-hydration water.

\section{Methods of Pore-Water Extraction for $\delta D$ and $\delta^{18} \mathrm{O}$ and Analysis}

It is important that a pore-water extraction method extract only the hydrologically active water (percolating mobile water), or the stable-isotope data of the extracted water will be misinterpreted. Extraction methods using vacuum distillation and toluene azeotropic distillation to remove water from soil and clay-rich soil have been reported by Revesz and Woods (1990), Walker and others (1994), and Ingraham and Shadel (1992). However, none had been applied to tuffaceous volcanic rocks. The distribution of minerals at Yucca Mountain differs from borehole to borehole, but certain commonalities are apparent. The major mineral groups of Yucca Mountain are zeolites, clays, alkali feldspar, silica polymorphs, and glass. The zeolites are primarily clinoptilolite, mordenite, and analcime. The clays are principally smectites and illite with lesser kaolinite (Broxton and others, 1986; Bish and Vaniman, 1985; Bish and Chipera, 1989). Zeolite and clay contain water of crystallization that can be released during the heatdistillation process. Three extraction methods toluene distillation, vacuum distillation, and compression - were used on core samples of volcanic rocks from Yucca Mountain in order to establish a standard extraction procedure.

\section{Toluene Distillation}

Toluene distillation of water from soil for isotopic analysis was first used by Allison and Hughes (1983). The principle of the toluene distillation method is that the toluene forms an azeotropic mixture with water at $84.1^{\circ} \mathrm{C}$ but separates from water at room temperature. The boiling point of the toluene-water azeotrope is $84.1^{\circ} \mathrm{C}$, significantly lower than the boiling point of water $\left(100^{\circ} \mathrm{C}\right)$ and toluene $\left(110^{\circ} \mathrm{C}\right)$. Ingraham and Shadel (1992) found that the isotopic compositions of water extracted during toluene distillation will fractionate when the distillation process is not completed. This result is predicted by the Rayleigh distillation model. The results indicate that the toluene is simply a carrier of water. Thus, fractionation will occur during the toluene distillation if the extraction is not complete.

The toluene distillation method used in this study is similar in design to that of Revesz and Woods (1990). The Dean-Staark apparatus used for toluene distillation (fig. 11) consists of a laboratory heating element, a flask, a specially designed receiving trap, and a straight condenser. The funnel has a wide area above the joint to prevent loss of water by uneven, explosive boiling (Revesz and Woods, 1990). Enough water-free toluene is added so that the sample remains covered throughout the entire distillation process. During distillation, the temperature is maintained at the boiling point $\left(84.1^{\circ} \mathrm{C}\right)$. The temperature of the solution is then raised to $110^{\circ} \mathrm{C}$. At the beginning of the distillation, water and toluene are distilled together, forming a cloudy liquid. The distillation continues until the condensate turns clear and no water 


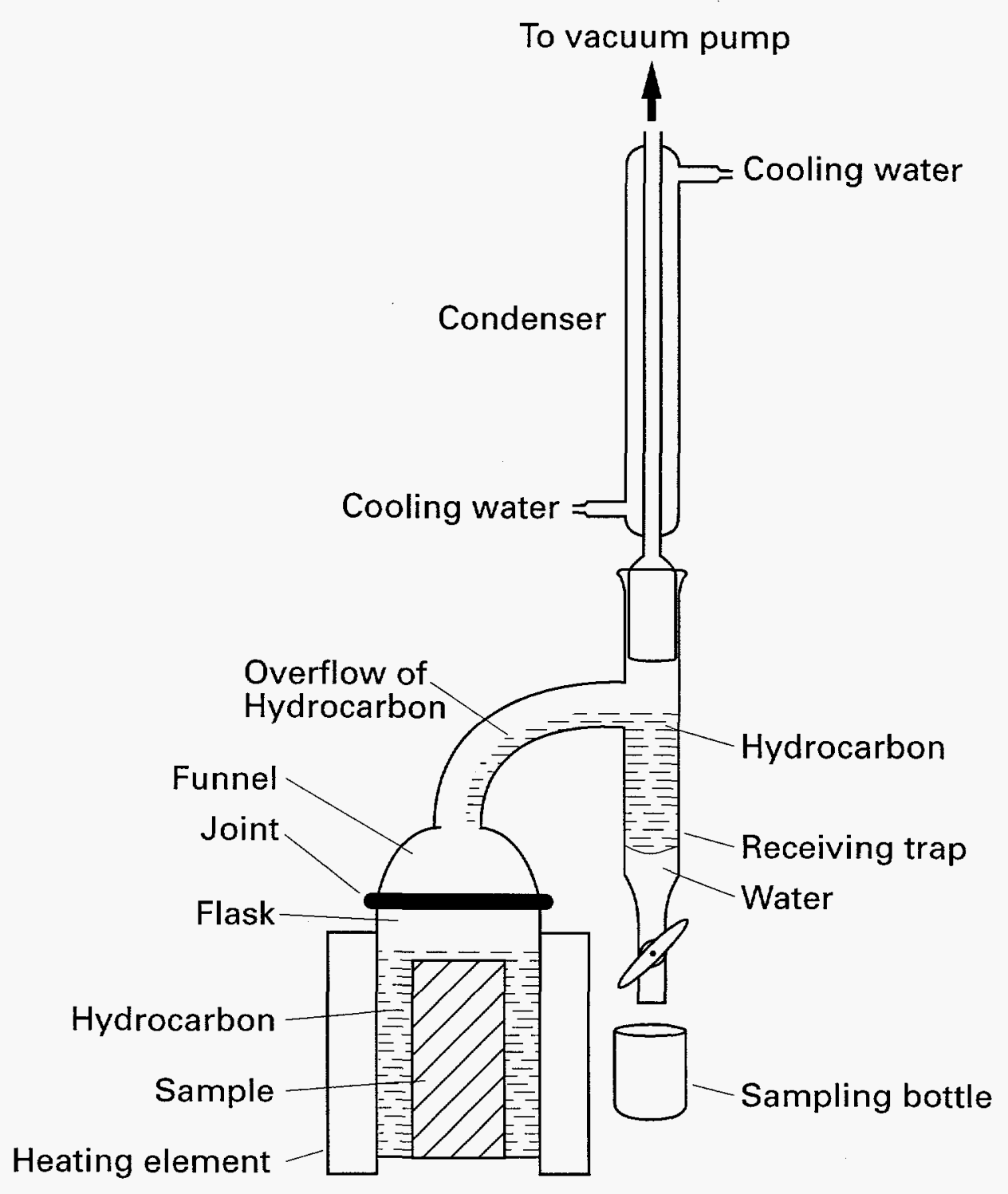

Figure 11. Toluene distillation apparatus for pore-water extraction.

drops remain on the glass wall of the entire apparatus. After collecting the water in a glass bottle with Teflon cap, any trace of toluene is removed by adding paraffin wax (enough to solidify after dissolving the toluene) to the water and warming it in the closed sample bottle to the melting point of the wax $\left(60-70^{\circ} \mathrm{C}\right)$. The bottle is shaken and allowed to cool, and the wax solidifies. The water is decanted easily. The wax procedure can be repeated to ensure complete removal of the toluene. The wax procedure was repeated three to four times in this study.

\section{Vacuum Distillation}

The vacuum distillation technique uses a distillation apparatus consisting of vacuum glassware, nitrogen gas, dry ice, a set of heating elements, and a vacuum pump (fig. 12). The procedure consists of four steps: (1) the core segment is weighed and placed inside the distillation apparatus; (2) a vacuum is applied to the distillation apparatus for a couple of hours and heated to $150^{\circ} \mathrm{C}$ (as measured at the heating mantle) resulting in a core temperature of about $110^{\circ} \mathrm{C}$ (the reason for choosing this temperature is explained below); (3) the core water is expelled into a flask cooled with a dry ice/alcohol mixture at a temperature of about $-80^{\circ} \mathrm{C}$; and (4) the total volume of water recovered is measured under nitrogen gas. The distilled core was placed inside an oven at $104^{\circ} \mathrm{C}$ for three days to check for any further loss of water. The third step may take around 24 hours to ensure complete extraction of core water. If the core water yield is less than 99.75 percent of the total measured water in the subsample (a small piece of core chipped 


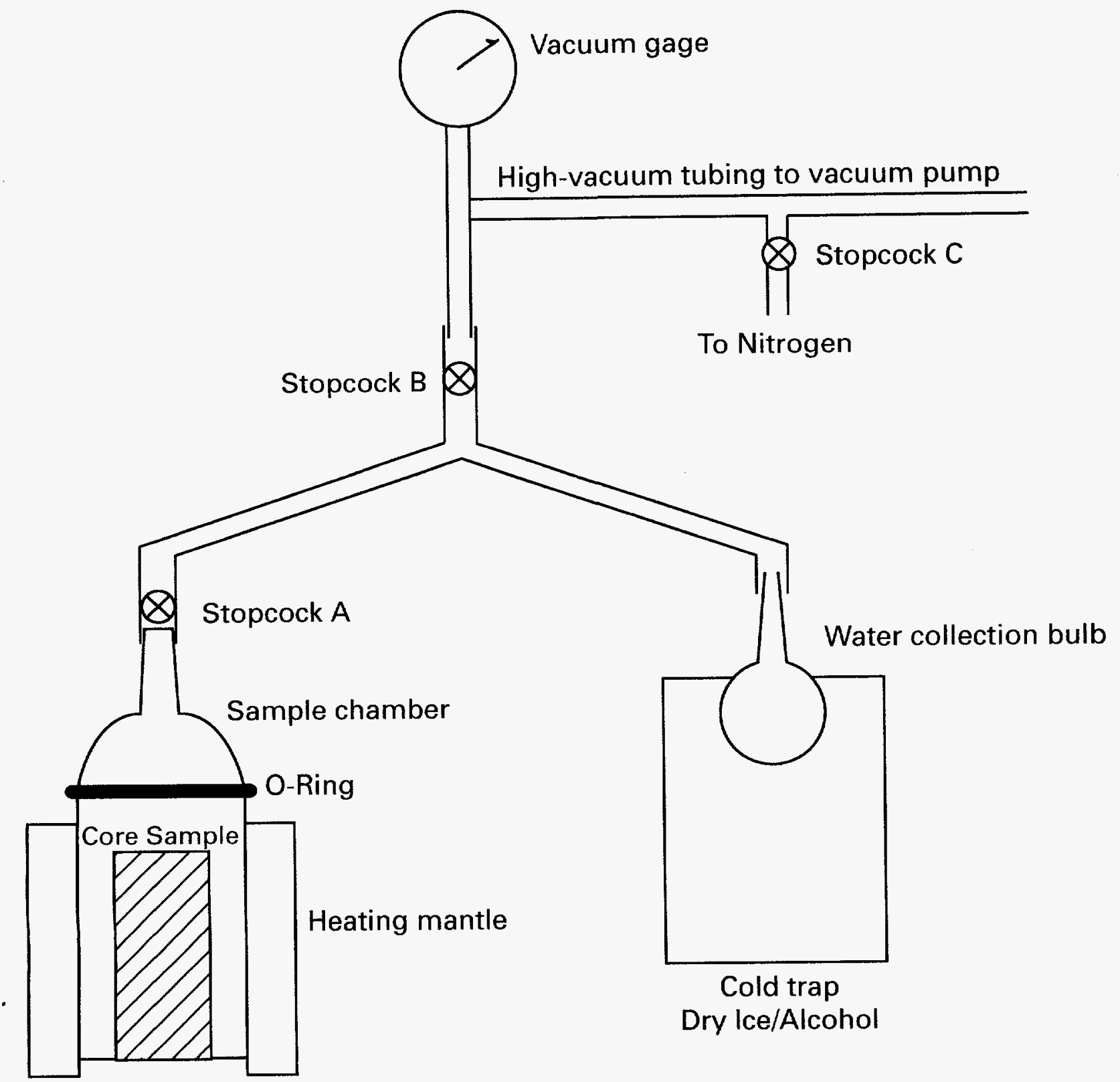

Figure 12. Vacuum distillation apparatus for pore-water extraction.

off and dried in the oven at $105^{\circ} \mathrm{C}$ to measure water content), there can be an appreciable amount of fractionation (Knowlton and others, 1989). If incomplete extraction was suspected, an additional core was vacuum-distilled for a longer time to make sure the water yield was greater than 99.75 percent.

The distillation temperature of the core sample will affect (1) the release of hydrated water, (2) the water-rock interaction at high temperature, and (3) the efficiency of extraction. Hydration water may mix with the pore water, thus masking the values of stable isotopes of pore water. The exchange of ${ }^{18} \mathrm{O}$ between water and rock at high temperature (greater than $150^{\circ} \mathrm{C}$ ) will enrich the ${ }^{18} \mathrm{O}$ of water (Panichi and Gonfiantini, 1981). Extraction efficiency increases with distillation temperature, but high temperature will cause problems from release of hydrated water and/or water-rock interaction. For clay-bearing soil, the temperature at which hydrated water is released is about $110^{\circ} \mathrm{C}$, as reported by Ingraham and Shadel (1992). They postulated that the hydration water of clay soils may not be released at temperatures below 
$110^{\circ} \mathrm{C}$; thus, an extraction method with a low temperature may be advantageous. Araguàs-Araguàs and others (1995) concluded that if the isotopic compositions of mobile water are of interest, the distillation temperature should be kept as low as possible to minimize the impact of removing the weakly bound hydration water. Feng and Savin (1993) found that some zeolite species (clinoptilolite, heulandite, and stilbite) will lose about 50 percent of hydrated (channel) water during overnight evacuation. Hydrated water is held in the structural channels of those minerals. However, the exchange of framework oxygen with bulk-water oxygen in zeolite was less than 5 percent at temperatures below $110^{\circ} \mathrm{C}$. For low-temperature distillation, Wood (1990) used vacuum distillation at room temperature, but the extraction was very poor because of incomplete recovery of water.

The distillation temperature for this study therefore was chosen at $150^{\circ} \mathrm{C}$ at the heating mantle, resulting in a core temperature of about $110^{\circ} \mathrm{C}$. From time-series measurements of mantle temperature and core temperature for different lithologic cores, the temperature distribution indicates that the temperature of the core increased with the temperature of mantle and equilibrated to core temperature of about $110^{\circ} \mathrm{C}$ when the heating-mantle temperature was at $150^{\circ} \mathrm{C}$ (Yu, 1996).

The time required for complete distillation of soil samples was reported by Ingraham and Shadel (1992) and Araguàs-Araguàs and others (1995) to be about 7 hours. However, other factors affect the time required for complete extraction, such as sample size, moisture, distillation temperature, and distillation system design. The optimum length of time for distillation was determined experimentally by comparing the stable-isotopic compositions of water distilled from a piece of selected Yucca Mountain core cut into five sections that were distilled for different time periods $(4,8,12,24$, and 36 hours). Five different lithologic cores were selected for the test-one core from the Pah Canyon Tuff, three cores from the Topopah Spring Tuff, and one core from the Calico Hills nonwelded hydrogeologic unit (table 6). The minimum distillation times are different, depending on the lithology. For Pah Canyon and Topopah Spring Tuff cores, at least 24 hours are required for complete extraction, but for Calico Hills nonwelded unit cores, more than 36 hours are required.

\section{Compression Extraction}

One-dimensional compression extraction (Higgins and others, 1997) was also tested. Compression operations were similar to the procedures described previously for pore-water extraction for chemical compositions and ${ }^{14} \mathrm{C}$ analyses. Water samples were collected in glass syringes attached to the compression cell when an adequate volume was extracted. The extracted water was filtered through a $0.45-\mu \mathrm{m}$ filter into glass bottles for stable-isotope analysis. The effect of the compression process on the alteration of original stable-isotope compositions of pore water can be investigated by two methods: (1) water samples are collected at each pressure level during the compression procedure, and stable-isotope compositions at each pressure level are then compared; and (2) a water of known stable-isotope composition is imbibed into dry cores (from each tuff) after the core was oven-dried overnight. The isotopic values of known water can be compared with those of the water extracted then from the imbibed cores by the compression method. If there is no change in composition as a function of pressure in the first test, this would indicate that the compression method did not cause pore-water fractionation. The results of the second test may indicate which pool of water inside the core is extracted by the compression method. If there is no difference in isotopic composition between known water and compression-extracted water, this would also indicate that compression processes did not alter the original pore-water isotopic compositions. On the other hand, if the stable-isotope composition of compression-extracted water is different from the imbibed water, and the extracted water does not change with pressure, the possible explanation is that the water imbibed into the dried core has been fractionated. That can be investigated by comparing the stable-isotope compositions of residual water with those of compression-extracted water.

\section{Water Analyses for $\delta^{18} \mathrm{O}$ and $\delta \mathrm{D}$}

Stable-isotope ratios $\delta^{18} \mathrm{O}$ and $\delta \mathrm{D}$ were analyzed using mass spectrometers at the University of Colorado, Institute of Arctic and Alpine Research Stable Isotope Laboratory and at the U.S. Geological Survey Research Laboratory in Reston, Virginia. The $\delta^{18} \mathrm{O}$ values were determined by isotopically equilibrating water with a small amount of pure $\mathrm{CO}_{2}$ in a constant-temperature bath. The equilibrated $\mathrm{CO}_{2}$ gas 
Table 6. Stable-isotopic compositions and water yield of water distilled from Yucca Mountain cores by use of different distillation times

$\left[\delta^{18} \mathrm{O}\right.$, delta oxygen-18; $\delta \mathrm{D}$, delta deuterium; VSMOW, Vienna Standard Mean Ocean Water; NA, not analyzed. There was not enough sample to perform oxygen- 18 analyses]

\begin{tabular}{|c|c|c|c|c|}
\hline $\begin{array}{c}\text { Step } \\
\text { number }\end{array}$ & $\begin{array}{c}\text { Collection time } \\
\text { (hours) }\end{array}$ & $\begin{array}{l}\text { Water yleld } \\
\text { (percent) }\end{array}$ & $\begin{array}{c}\delta^{18} \mathrm{O} \\
\text { relative to } \\
\text { VSMOW } \\
\text { (per mil) }\end{array}$ & $\begin{array}{c}\delta D \\
\text { relative to } \\
\text { VSMOW } \\
\text { (per mll) }\end{array}$ \\
\hline \multicolumn{5}{|c|}{ Run A. Core ID: UZ14-156.2 from Pah Canyon Tuff } \\
\hline 1 & 4 & 10.12 & -15.1 & -115 \\
\hline 2 & 8 & 15.12 & -13.2 & -104 \\
\hline 3 & 12 & 18.33 & -12.9 & -99 \\
\hline 4 & 24 & 19.78 & -12.2 & -96 \\
\hline 5 & 36 & 19.82 & -12.1 & -96 \\
\hline \multicolumn{5}{|c|}{ Run B. Core ID: UZ14-1020 from Topopah Spring Tuff } \\
\hline 1 & 4 & 3.42 & -14.9 & -110 \\
\hline 2 & 8 & 4.51 & -14.2 & -104 \\
\hline 3 & 12 & 5.20 & -13.3 & -99 \\
\hline 4 & 24 & 5.21 & -13.6 & -99 \\
\hline 5 & 36 & 6.03 & -13.4 & -99 \\
\hline 6 & 48 & 6.03 & -13.2 & -98 \\
\hline \multicolumn{5}{|c|}{ Run C. Core ID: UZ14-1187.8 from Topopah Spring Tuff } \\
\hline 1 & 4 & 2.16 & NA & -106 \\
\hline 2 & 8 & 3.71 & -16.3 & -103 \\
\hline 3 & 12 & 3.96 & -16.5 & -103 \\
\hline 4 & 24 & 4.08 & -15.0 & -102 \\
\hline 5 & 36 & 4.08 & -15.1 & -102 \\
\hline \multicolumn{5}{|c|}{ Run D. Core ID: UZ14-1391.2 from Topopah Spring Tuff } \\
\hline 1 & 4 & 11.13 & -16.2 & -112 \\
\hline 2 & 8 & 17.55 & -16.7 & -109 \\
\hline 3 & 12 & 18.43 & -16.5 & -108 \\
\hline 4 & 24 & 21.90 & -16.7 & -109 \\
\hline 5 & 36 & 21.90 & -16.7 & -109 \\
\hline \multicolumn{5}{|c|}{ Run E. Core ID: UZ14-1430.2 from Calico Hills nonwelded hydrogeologic unit } \\
\hline 1 & 4 & 4.28 & -19.1 & -140 \\
\hline 2 & 8 & 16.67 & -16.3 & -112 \\
\hline 3 & 12 & 17.35 & -16.2 & -108 \\
\hline 4 & 24 & 18.53 & -16.0 & -109 \\
\hline 5 & 36 & 18.50 & -15.9 & -106 \\
\hline 6 & 48 & 18.51 & -15.8 & -107 \\
\hline
\end{tabular}

was measured in the mass spectrometer, and the $\delta^{18} \mathrm{O}$ of the water was calculated from this value. Typically $4 \mathrm{~mL}$ of water are used, although smaller samples (down to $2 \mathrm{~mL}$ ) can be measured in this way. The equilibration system is automated and controlled by a computer that also controls the operation of the mass spectrometer. All critical parameters of the system (pressures and temperatures) are monitored by the computer, and any sample that experiences conditions outside of acceptable ranges is rejected. The $\delta^{18} \mathrm{O}$ equilibration system is capable of measuring 20 samples and 4 standards overnight.

The $\delta \mathrm{D}$ values were determined by reducing water to hydrogen gas and by measuring the $\delta \mathrm{D}$ of the hydrogen in the mass spectrometer. Water was injected into a hot evacuated cell. The water vapor thus formed was passed through a capillary tube and over uranium metal at $600^{\circ} \mathrm{C}$. The uranium metal reduced water 
vapor to hydrogen gas. The injection of water, reduction to hydrogen, and measurement of $\delta \mathrm{D}$ values in the mass spectrometer are all controlled by computer. The volume of water required is $2 \mathrm{~mL}$. This system is capable of measuring 20 samples and 6 standards in about 20 hours. Samples prepared for this method should have a conductivity of $2,000 \mu \mathrm{S} / \mathrm{cm}$ or less to avoid salt buildup in the evacuated cell. Uncertainty in $\delta^{18} \mathrm{O}$ values was 0.2 per mil and in $\delta \mathrm{D}$ was 1.0 per mil. These uncertainties are one standard deviation $(1 \sigma)$. Relative ${ }^{18} \mathrm{O} /{ }^{16} \mathrm{O}\left(\delta^{18} \mathrm{O}\right)$ and $\mathrm{D} / \mathrm{H}(\delta \mathrm{D})$ values of water expressed in \%o relative to VSMOW water $\left(0 \%\right.$ ) on a scale normalized so that the $\delta^{18} \mathrm{O}$ and $\delta \mathrm{D}$ of Standard Light Antarctic Precipitation (SLAP) water are $-55.5 \%$ and $-428 \%$, respectively.

\section{Inconsistency in $\delta \mathrm{D}$ and $\delta^{18} \mathrm{O}$ Data with Different Extraction Methods}

The extraction of isotopically representative water from porous media for stable-isotope analysis has been sometimes difficult, particularly in the case of unsaturated core, in which not all pore spaces are filled with water. It is mandatory that no fractionation (change in isotopic composition) occurs during the extraction processes. Pore water extracted from samples from the same piece of core by different methods ideally should yield the same isotopic composition.

\section{Review of Water-Extraction Methods Published in the Literature}

Walker and others $(1992,1994)$ compared the stable-isotope data from 14 different laboratories on four continents for soil water derived by various extraction methods. They distributed soils rehydrated with water of known stable-isotope composition to the 14 laboratories where the soil water was extracted and returned for analysis. Extraction methods included toluene-azeotropic distillation (six laboratories), vacuum distillation (seven laboratories), microdistillation (three laboratories), and centrifugation (one laboratory). The comparison showed large variation among laboratories in isotopic compositions of the extracted water (up to 30 per mil for $\delta \mathrm{D}$ and 4.4 per mil for $\delta^{18} \mathrm{O}$ ). The measured compositions from extracted waters were commonly lighter than those of the introduced water by more than 10 per mil in $\delta \mathrm{D}$ and 2 per mil in $\delta^{18} \mathrm{O}$. The variation increased as the water content of the soil decreased and was greater for clays than for sand at a comparable matrix suction force. The variation was caused by incomplete extraction of soil water from the soil samples. Centrifugation followed by toluene-azeotropic distillation gave the results closest to the known value.

Because few studies have examined extraction methods for stable-isotope analyses of hydrated mineral soil (soil containing certain clays or gypsum), Walker and others $(1992,1994)$ suggested that toluene-azeotropic distillation and vacuum distillation are unsuitable for gypsum media because gypsum is unstable above about $45^{\circ} \mathrm{C}$. At temperatures above $45^{\circ} \mathrm{C}$, gypsum releases its hydrated water to form bassanite, which results in the fractionation. Centrifugation and compression at room temperature may be useful but are generally restricted to samples of high water content only.

Other studies examined these problems. Ingraham and Shadel (1992) did a comparative study of the toluene and vacuum distillation methods using Hanford loam soil. The stable-isotope compositions of water extracted by these two methods were significantly different from those of the added water when the water content of the soil was low (3.6 to 5.2 percent). They postulated that some of the added water bound with minerals in the soil with an associated fractionation and was not released at extraction temperatures below $110^{\circ} \mathrm{C}$. At low soil-moisture content, the volume of fractionated bound water is enough to affect the remaining extracted soil water. Leaney and others (1993) used the azeotropic distillation method with kerosene as a solvent for soils with high clay content ( 20 to 60 percent). They found a large discrepancy in the $\delta \mathrm{D}$ composition between added water and extracted water. The extracted water was 3 to 10.8 per mil more depleted in deuterium than the added water, depending on the temperature of distillation and the content of clay. Only deuterium was measured in that experiment. When extrapolated to zero percent clay content, the extracted water was 3 per mil more depleted than added water. They concluded that fractionation likely occurred during solvent extraction of soil and that the extracted water would be depleted by more than 3 to 4 per mil in $\delta D$ compared with the true value of soil water. AraguàsAraguàs and others (1995) performed the vacuumdistillation method on clay-bearing soil. The data indicated that the extracted water was depleted in 
deuterium by about 5 to 10 per mil and 0.36 per mil in oxygen, when compared with mobile water.

\section{Analytical Data from Methods Used in This Study}

Eleven sections of cores, three from nonwelded bedded tuff, one from the Pah Canyon Tuff, four from the Calico Hills nonwelded unit, and three from the Prow Pass Tuff, were chosen to determine if there is any alteration of the stable-isotope compositions of pore water extracted by toluene-azeotropic distillation, vacuum distillation, and compression extraction. Clay or zeolite minerals occur in the nonwelded bedded tuff and the Calico Hills nonwelded unit but not in the Pah Canyon Tuff. Cores from the Prow Pass Tuff, from depths of $550.0 \mathrm{~m}$ and $625.1 \mathrm{~m}$, contain zeolite, but core from a depth of $564.2 \mathrm{~m}$ does not. A comparison of data from the Topopah Spring Tuff was not possible because the water content of this formation is too low to provide enough water for analysis by the compression method.

\section{Isotopic Data from Toluene-Azeotropic Distillation}

Five sections of cores from Yucca Mountain Tuff, Pah Canyon Tuff, Topopah Spring Tuff, Calico Hills Formation, and Prow Pass Tuff were imbibed with water of known isotopic composition. The source water was Boulder, Colorado, tap water with an isotopic composition of -17.7 per mil for $\delta^{18} \mathrm{O}$ and -129 per mil for $\delta \mathrm{D}$. The imbibed cores were then subjected to toluene distillation to extract water for stable-isotope analyses. Table 7 shows the stableisotope compositions of distilled water, water recovery, and major mineralogy of cores. The data indicate that the isotopic compositions of recovered water were more depleted than the source water by 1.9 to 3.7 per mil for $\delta^{18} \mathrm{O}$ and 9 to 23 per mil for $\delta \mathrm{D}$. The water recoveries are much less than 100 percent, indicating incomplete recovery of core water by toluene distillation. Small water yields may be due to the use of intact core instead of crushed core, which would yield more surface area for water distillation. Based on the observation of Ingraham and Shadel (1992), the variation of the stable isotopes may be caused by incomplete extraction. From the results of the imbibition tests, the toluene distillation method is evidently unsuitable for extracting water from the Yucca Mountain cores and is not considered further in this report.

\section{Comparison of Vacuum-Distillation and Compression-Extraction Methods}

In a comparison test, eleven sections of dried core were chosen to test effects of the vacuum-distillation and compression-extraction methods on the measured stable-isotope compositions of in-situ pore water. Different samples were used than for the toluene extraction (table 7), but samples covered a similar lithologic range (three from bedded tuff, one from the Pah Canyon Tuff, four from the Calico Hills nonwelded hydrogeologic unit, and three from the Prow Pass Tuff). Table 8 shows mineralogic data from the samples chosen for the vacuum-distillation and compression-extraction tests.

In the bedded tuff (table 9), three cores from depths of about 26 to $29 \mathrm{~m}$ indicate that the stableisotope compositions of the water collected by the compression method are heavier than the remaining water (water extracted by vacuum distillation after compression) in the range of 5 to 14 per mil for $\delta \mathrm{D}$ and 0.2 to 1.9 per mil for $\delta^{18} \mathrm{O}$. The compressed water also is more enriched than water extracted from samples from nearby core by vacuum distillation in the range of 6 to 9 per mil for $\delta \mathrm{D}$ and 0.1 to 1.1 per mil for $\delta^{18} \mathrm{O}$. The stable-isotope compositions of compressed water collected at different pressure steps during the compression process changed little with pressure changes. Mineralogic data indicate that all of the bedded tuff cores contain clay minerals.

The tested cores of Pah Canyon Tuff from a depth of $49 \mathrm{~m}$ (table 10) indicate that the stableisotope compositions of water collected by different methods are not substantially different. The stableisotope compositions of the water collected at different pressure steps during the compression process are similar, with little variation as a function of pressure. These data imply that both distillation and compression methods have no effect on the stable-isotope compositions of pore water for cores from Pah Canyon Tuff, which contain few hydrated minerals and which especially lack zeolites (table 8).

Three cores were sampled from the depth interval of $446.7 \mathrm{~m}$ to $476.9 \mathrm{~m}$ from the Calico Hills nonwelded hydrogeologic unit (table 11). Mineralogic data (table 8) indicate that these cores contain abundant zeolitic minerals. The stable-isotope compositions of the water collected by the compression method are heavier relative to the remaining water extracted by vacuum distillation in the range of 2.6 to 
Table 7. Results of the toluene-distillation method in extracted water from imbibed cores, Yucca Mountain area

[VSMOW, Vienna Standard Mean Ocean Water; source water, water with known isotopic composition used for imbibing test; \%, percent]

\begin{tabular}{|c|c|c|c|}
\hline $\begin{array}{l}\text { Sample identification, lithology, and mineralogy } \\
\text { Lithology (Mineralogy) }\end{array}$ & $\begin{array}{l}\delta^{18} 0 \\
\text { relative to } \\
\text { VSMOW } \\
\text { (per mil) }\end{array}$ & $\begin{array}{l}\delta D \\
\text { relative to } \\
\text { VSMOW } \\
\text { (per mil) }\end{array}$ & $\begin{array}{l}\text { Water } \\
\text { recovery } \\
\text { (percent) }\end{array}$ \\
\hline UZ14-77.4/T16/To & -21.3 & -152 & 60.5 \\
\hline \multicolumn{4}{|c|}{ Yucca Mountain Tuff: Amorphous 30-35\%, montmorillonite $14 \%$, plagioclase $20 \%$} \\
\hline UZ14-215/T16/To & -19.6 & -138 & 80.2 \\
\hline \multicolumn{4}{|l|}{ Pah Canyon Tuff: Mineralogic data not available } \\
\hline UZ14-1271.7/T16/To & -21.4 & -149 & 52.1 \\
\hline \multicolumn{4}{|c|}{ Topopah Spring Tuff: Plagioclase 34\%, K-spar 20\%, Amorphous material 10-15\% } \\
\hline UZ14-1514.2/T16/To & -20.5 & -141 & 67.8 \\
\hline \multicolumn{4}{|l|}{ Calico Hills Formation: Amorphous $15-20 \%$, Zeolite $67 \%$} \\
\hline UZ14-1943.6/T16/To & -19.7 & -140 & 60.7 \\
\hline \multicolumn{4}{|l|}{ Prow Pass Tuff: Zeolite $28 \%$, Plagioclase $11 \%$, Amorphous material $25-30 \%$ ) } \\
\hline Source water & -17.7 & -129 & \\
\hline
\end{tabular}

Table 8. Mineralogic data from Yucca Mountain core samples used for comparing the vacuum-distillation and compression-extraction methods for stable-isotope analysis

\begin{tabular}{|c|c|c|c|c|c|c|}
\hline $\begin{array}{c}\text { Sample } \\
\text { identification }\end{array}$ & $\begin{array}{c}\text { Zeolite } \\
\text { (percent) }\end{array}$ & $\begin{array}{c}\text { Clay } \\
\text { (percent) }\end{array}$ & $\begin{array}{c}\text { Plagioclase } \\
\text { (percent) }\end{array}$ & $\begin{array}{l}\text { Amor- } \\
\text { phous } \\
\text { material } \\
\text { (percent) }\end{array}$ & $\begin{array}{c}\text { Other } \\
\text { (percent) }\end{array}$ & Lithology \\
\hline UZ14-85.2 & 0 & 56 & 5 & $30-35$ & $4-9$ & Bedded tuff \\
\hline UZ14-91.0 & 0 & 30 & 20 & $20-30$ & $20-30$ & Bedded tuff \\
\hline UZ14-95.5 & 0 & 30 & 20 & $20-30$ & $20-30$ & Bedded tuff \\
\hline UZ14-155.7 & 0 & 0 & 30 & $30-35$ & $35-40$ & Pah Canyon Tuff \\
\hline UZ14-1465.5 & 35 & 0 & 10 & $30-35$ & $20-25$ & $\begin{array}{l}\text { CHn Calico Hills } \\
\text { nonwelded uni }\end{array}$ \\
\hline UZ14-1542.3 & 35 & 0 & 10 & $30-35$ & $20-25$ & $\begin{array}{l}\mathrm{CHn} \text { Calico Hills } \\
\text { nonwelded uni }\end{array}$ \\
\hline UZ14-1564.3 & 35 & 0 & 10 & $30-35$ & $20-25$ & $\begin{array}{l}\mathrm{CHn} \text { Calico Hills } \\
\text { nonwelded uni }\end{array}$ \\
\hline UZ14-1734.5 & 32 & 0 & 15 & $20-30$ & $33-43$ & $\begin{array}{l}\mathrm{CHn} \text { Calico Hills } \\
\text { nonwelded uni }\end{array}$ \\
\hline UZ14-1804.8 & 0 & 56 & 5 & $30-35$ & $4-9$ & Prow Pass Tuff \\
\hline UZ14-1854.8 & 0 & 0 & 30 & $5-10$ & $60-85$ & Prow Pass Tuff \\
\hline UZ14-2015.2 & 28 & 0 & 11 & $25-30$ & $21-26$ & Prow Pass Tuff \\
\hline
\end{tabular}


Table 9. Stable-isotope compositions of compressed water (IUP), remaining water extracted by vacuum distillation (UP/D), and water distilled (ID) from bedded tuff cores

[Remaining water, water remaining in the core after compression; distilled water; the whole-core water extracted only by vacuum distillation; VSMOW, Vienna Standard Mean Ocean Water]

\begin{tabular}{lcc}
\hline Sample identification & $\begin{array}{c}\delta^{18} \circ \\
\text { relative to } \\
\text { vSMOW } \\
\text { (per mil) }\end{array}$ & $\begin{array}{c}\delta D \\
\text { relative to } \\
\text { VSMOW } \\
\text { (per mil) }\end{array}$ \\
\hline UZ14-85.2-85.6/UP1 & -12.7 & -90 \\
UZ14-85.2-85.6/UP2 & -12.4 & -89 \\
UZ14-85.2-85.6/UP5 & -12.6 & -91 \\
UZ14-85.2-885.6/UP6 & -12.7 & -91 \\
UZ14-85.2-85.6/UP/D & -13.2 & -103 \\
UZ14-84.3-65.6/D & -12.8 & -97 \\
& & \\
UZ14-91.0-91.4/UP & -12.4 & -90 \\
UZ14-91.0-91.4/UP/D1 & -13.9 & -103 \\
UZ14-91.0-91.4/UP/D2 & -14.3 & -104 \\
UZ14-89.6-91.4/D & -13.5 & -97 \\
& & \\
UZ14-95.5-95.9/UP1 & -12.4 & -90 \\
UZ14-95.5-95.9/UP2 & -12.3 & -90 \\
UZ14-95.5-95.9/UP3 & -12.3 & -89 \\
UZ14-95.5-95.9/UP4 & -12.7 & -91 \\
UZ14-95.5-95.9/UP/D & -13.6 & -103 \\
UZ14-94.8-95.9/D & -13.4 & -98 \\
\hline
\end{tabular}

Table 10. Stable-isotopic compositions of compressed water (UP) and water distilled (/D) from the Pah Canyon Tuff cores

[VSMOW, Vienna Standard Mean Ocean Water]

\begin{tabular}{lcc}
\hline Sample identification & $\begin{array}{c}\delta^{\mathbf{1 8}} \mathbf{0} \\
\text { (per mil) } \\
\text { relative to } \\
\text { VSMOW }\end{array}$ & $\begin{array}{c}\delta \mathbf{D} \\
\text { (per mil) } \\
\text { relative to } \\
\text { VSMOW }\end{array}$ \\
\hline UZ14-155.7-156.2/UP1 & Core ID: UZ14-155.7-156.2 & -88 \\
UZ14-155.7-156.2/UP2 & -12.5 & -88 \\
UZ14-155.7-156.2/UP3 & -11.7 & -88 \\
UZ14-155.7-156.2/UP4 & -11.8 & -88 \\
UZ14-155.7-156.2/D & -11.6 & -88 \\
\hline
\end{tabular}


Table 11. Stable-isotope compositions of compressed water (IUP), remaining water extracted by vacuum distillation (UPP/D), and water distilled (/D) from the Calico Hills Formation cores

[VSMOW; Vienna Standard Mean Ocean Water]

\begin{tabular}{|c|c|c|}
\hline Sample identification & $\begin{array}{c}\delta^{18} \mathrm{O} \\
\text { (per mil } \\
\text { relative to } \\
\text { VSMOW }\end{array}$ & $\begin{array}{c}\delta \bar{D} \\
\text { (per mil) } \\
\text { relative to } \\
\text { VSMOW }\end{array}$ \\
\hline \multicolumn{3}{|c|}{ Core ID: UZ14-1455.5-1466.1 } \\
\hline UZ14-1465.5-1466.1/UP2 & -13.6 & -102 \\
\hline UZ14-1465.5-1466.1/UP3 & -13.6 & -102 \\
\hline UZ14-1465.5-1466.1/UP4 & -13.5 & -102 \\
\hline UZ14-1465.5-1466.1/UP5 & -13.6 & -102 \\
\hline UZ14-1465.5-1466.1/UP6 & -13.6 & -102 \\
\hline UZ14-1465.5-1466.1/UP7 & -13.6 & -102 \\
\hline UZ14-1465.5-1466.1/UP/D & -16.1 & -121 \\
\hline UZ14-1465.5-1466.1/D & -15.9 & -111 \\
\hline \multicolumn{3}{|c|}{ Core ID: UZ14-1542.3-1542.7 } \\
\hline UZ14-1542.3-1452.4/UP & -13.4 & -100 \\
\hline UZ14-1542.3-1452.4/UP/D & -16.1 & -117 \\
\hline \multicolumn{3}{|c|}{ Core ID: UZ14-1564.3-1564.7 } \\
\hline UZ14-1564.3-1564.7/UP & -13.7 & -103 \\
\hline UZ14-1564.3-1564.7/UP/D & -20.0 & -125 \\
\hline UZ14-1564.3-1564.7/D & -18.0 & -114 \\
\hline
\end{tabular}

6.3 per mil for $\delta^{18} \mathrm{O}$ and 17 to 22 per mil for $\delta \mathrm{D}$. The compressed waters also are heavier than the water extracted from the adjacent core by vacuum distillation in the range of 2.3 to 4.3 per mil for $\delta^{18} \mathrm{O}$ and 9 to 11 per mil for $\delta \mathrm{D}$. The stable-isotope compositions of the compressed water collected at different pressure steps during the compression process are similar. As with the bedded tuff samples (table 9), the differences in stable-isotope compositions may be attributed to extraction of isotopically depleted water from hydrated minerals during the distillation process.

Three cores also were sampled from the Prow Pass Tuff from depths of $550.0 \mathrm{~m}$ to $614.3 \mathrm{~m}$ (table 12). One core contains zeolitic minerals (UZ14-2015.2), and one does not (UZ14-1854.8). For the clay-bearing core, the stable-isotope composition of compressed water is heavier than the remaining water by 20 per mil for $\delta \mathrm{D}$ and 4.1 per mil for $\delta^{18} \mathrm{O}$ and also is heavier than water extracted by whole-core vacuum distillation by 13 to 16 per mil for $\delta \mathrm{D}$ and 3.2 to 5.8 per mil for $\delta^{18} \mathrm{O}$. For the non-zeolite and nonclay-bearing core (UZ14-1854.8), the stableisotope compositions of compressed water are similar to the distilled water. These data indicate that if core samples contain zeolites, distilled water and compressed water will have different $\delta \mathrm{D}$ and $\delta^{18} \mathrm{O}$ values.

Table 12. Stable-isotope compositions of compressed water (UP), remaining water extracted by vacuum distillation (UUP/D), and water distilled (ID) from the Prow Pass Tuff cores [VSMOW, Vienna Standard Mean Ocean Water]

\begin{tabular}{ccc}
\hline Sample identification & $\begin{array}{c}\delta^{18} \mathbf{0} \\
\text { (per mil) } \\
\text { relative to } \\
\text { VSMOW }\end{array}$ & $\begin{array}{c}\delta D \\
\text { (per mil) } \\
\text { relative to } \\
\text { vSMOW }\end{array}$ \\
\hline Core ID: UZ14-1804.8-1805.3 & \\
UZ14-1804.8-1805.3/UP & -13.1 & -98 \\
UZ14-1804.8-1805.3/UP/D & -17.2 & -118 \\
UZ14-1804.8-1805.3/D & -16.3 & -111 \\
Core ID: UZ14-1854.8-1855.3 & \\
UZ14-1854.8-1855.3/UP & -13.3 & -100 \\
UZ14-1854.4-1854.8/D & -13.4 & -101 \\
Core ID: UZ14-2015.2-2015.6 & \\
UZ14-2015.2-2015.6/UP & -12.5 & -99 \\
UZ14-2014.0-2014.4/D & -18.3 & -115 \\
\hline
\end{tabular}

